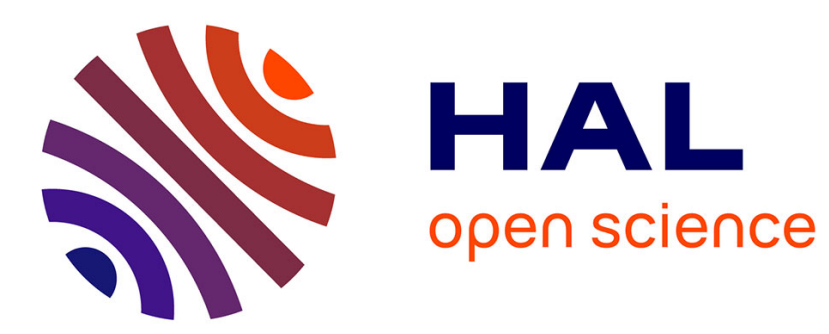

\title{
Coalition configurations and share functions
}

Nicolas G. Andjiga, Sébastien Courtin

\section{To cite this version:}

Nicolas G. Andjiga, Sébastien Courtin. Coalition configurations and share functions. Annals of Operations Research, 2015, 225 (1), pp.3 - 25. 10.1007/s10479-014-1754-8 . hal-00914883

\section{HAL Id: hal-00914883 \\ https://hal.science/hal-00914883}

Submitted on 9 Dec 2013

HAL is a multi-disciplinary open access archive for the deposit and dissemination of scientific research documents, whether they are published or not. The documents may come from teaching and research institutions in France or abroad, or from public or private research centers.
L'archive ouverte pluridisciplinaire $\mathbf{H A L}$, est destinée au dépôt et à la diffusion de documents scientifiques de niveau recherche, publiés ou non, émanant des établissements d'enseignement et de recherche français ou étrangers, des laboratoires publics ou privés. 


\title{
Coalition configurations and share functions
}

\author{
Nicolas G. Andjiga* $\quad$ Sebastien Courtin ${ }^{\dagger}$
}

October 2012

\begin{abstract}
Albizuri et al. (2006a, 2006b) defined values for games in which the players are organized into an a priori coalition configuration. In games with coalition configuration, we suppose that players organize themselves into coalitions that are not necessarily disjoint. A player can belong to more than one a priori coalition. In this paper we redefine coalition configuration values by using the concept of share function, as introduced by van der Laan and van den Brink (1998). A share function assigns to every player in a game its share in the worth to be distributed. We also define and characterize a general class of share function for games with coalition configuration which contains among other values those introduced by Albizuri et al. (2006a, 2006b).
\end{abstract}

Jel classification: C71

Keywords: Coalition configuration, Coalition structure, Share function, Shapley value, Banzhaf value.

\section{Introduction}

A cooperative game with transferable utility (TU-game) describes a situation in which players can obtain a payoff through cooperation. A value function for such a game is a function which assigns to every TU-game a distribution of payoffs among the players. One of the main solution concepts for TU-games is the Shapley value (1953). Another well-known solution is the Banzhaf value (Banzhaf (1965) and Owen (1975) for a generalization to all TU-games). The problem with these values is that they do not take into consideration a priori relations between different players. Indeed in many negotiations some agents prefer to cooperate with each other rather than with other players due to the existence of common interests. For example, consider international diplomatic relations, especially those of France and the United States. France belongs to the European Union (EU), which is an a priori coalition of European countries, whereas the USA belongs to an a priori coalition with Mexico and Canada (NAFTA). Classical values do not take into consideration this kind of relationship.

Some authors (see Aumann and Drèze 1974, Owen 1977 and 1981, among others) then introduced games in an a priori coalition structure. A coalition structure is a finite partition of the player set in disjoint coalitions. In such a game it is supposed that players organize themselves into coalitions that are necessarily disjoint. More formally the relationship between the players that specifies a partition of the set of players is an equivalence relation, that is, a reflexive, symmetric and transitive binary relation. ${ }^{1}$

Owen $(1977,1981)$ proposed and characterized a modification of the Shapley and the Banzhaf values with respect to a coalition structure. In this case there is a two-level interaction between players. Firstly, coalitions play an external game among themselves, and each one receives a payoff; secondly, in internal games the payoffs of each coalition are distributed amongst their members. Both payoffs, in the external game and in the internal game, are given by the Shapley value or the Banzhaf value.

But it is clear that all the relationships between players are not necessarily an equivalent relation. In real life there is no reason to assume that individuals defending their interests will organize themselves into coalitions that are necessarily disjoint. In other words, the relations between players need not to be transitive. It is well known that France belongs to more than one a priori international coalition. Among other things France belongs to the European Union, and to NATO.

\footnotetext{
*Ecole Normale Supérieure, Université de Yaoundé, B.P. 47, Yaoundé, Cameroon. Email:andjiga2002@yahoo.fr

${ }^{\dagger}$ Corresponding author. Université de Cergy-Pontoise, THEMA, UMR CNRS 8184, 95011 Caen, France. Email:sebastien.courtin@ucergy.fr. Tel: $(+33) 134252303$.

${ }^{1} \mathrm{~A}$ relation is: reflexive if every element in a set is related to itself; symmetric if it holds for each element a and $\mathrm{b}$ that if a is related to $\mathrm{b}$ then $\mathrm{b}$ is related to $\mathrm{a}$; and transitive if whenever an element $\mathrm{a}$ is related to an element $\mathrm{b}$, and $\mathrm{b}$ is in turn related to an element c, then a is also related to c.
} 
Consequently coalition structures do not adequately represent some bargaining situations. One solution for modeling these more complex relationships between different players is to consider the more general concept of coalition configuration, as introduced by Albizuri et al. (2006a, 2006b). Rather than considering disjoint coalitions in a game with coalition configuration, we consider the division of the individual sets into coalitions which are not necessarily disjoint, and whose union is the grand coalition. It is assumed that players can belong to more than one coalition so that they might obtain an improved bargaining position. Albizuri et al. (2006a, 2006b) generalized the Shapley and the Banzhaf values with reference to coalition configurations, and simultaneously the values proposed by Owen $(1977,1981)$.

Albizuri et al. (2006a, 2006b) provided interesting axiomatic characterizations for their values. However, as regards the Banzhaf value, the generalization that they present to games with coalition configuration fails to satisfy the efficiency axiom ${ }^{2}$. One way of solving this problem is to normalize this value in the same way that we can normalize the Banzhaf value. However, as pointed out by Dubey and Shapley (1979), such normalization is not as innocent as it seems. Indeed the normalized Banzhaf value violates two important axioms, namely the dummy player property and the additivity axiom ${ }^{3}$.

The concept of share function introduced by van der Laan and van den Brink (1998) is an alternative approach to efficiently allocating the worth of the grand coalition. A share function assigns to every player in a TU-game its share in the value to be distributed. In other words, the payoff of the grand coalition is completely redistributed to all players. They first applied the concept of share function to the Shapley and the Banzhaf values in 1998. The Shapley (or Banzhaf) share function assigns to every player its Shapley (Banzhaf) value divided by the sum of the Shapley (Banzhaf) values of all players in the game. Moreover they provided an axiomatic characterization of a class of share functions containing among others the Shapley share function and the Banzhaf share function.

In the same way, in 2002 and 2005, they introduced share functions for games with coalition structure. They again obtained an axiomatic characterization of a class of share functions for games with coalition structure containing the share functions corresponding to the values introduced by Owen as special case. The class of share functions for games with coalition structure presented in this paper will be slightly different from the class introduced by van den Brink and van der Laan (2005) (see Section 4).

We find two advantages in share functions. Firstly, beside the fact that a share function respects efficiency, the use of share functions has no impact under the other properties verified by the original values. And secondly, it is a natural and simple method to define a general class of share function.

In this paper we apply the idea of share functions, as introduced by van der Laan and van den Brink (1998), to define solutions for games with a coalition configuration. We first redefine the coalition configuration values corresponding to the Shapley value given by Albizuri et al. (2006a, 2006b) as a coalition configuration share function. This share function satisfies a variant of the multiplication property introduced by van der Laan and van den Brink (2002) and, van den Brink and van der Laan (2005). According to this property, the share of a player in the value of the grand coalition is equal for each coalition he belongs to, as the product of two shares: a share in the internal game and a share in the external game. Using this multiplication property, we generalize the Banzhaf share function to games with a coalition configuration.

Since the concept of share function also provides a natural method for defining solutions for games with a coalition configuration, we define a general class of share functions for games with coalition configuration. We characterize the new value by adapting van den Brink and van der Laan (2005) axioms, and adding a specific axiom called "merger" (see Albizuri et al. 2006a and 2006b). This axiom refers to a special sort of symmetric player, named doubles. The merger axiom requires that these doubles can be amalgamated into a single player without affecting the payoffs of the rest of players. This axiom characterizes, with another axiom, the coalition configuration values

The remainder of this paper is organized as follows. The next section contains some preliminaries on games and values, both for coalition structures and for coalition configurations. In Section 3 we recall the concept of share functions and present some results regarding games with coalition structure. In Section 4, we introduce a class of share functions for games with coalition configuration. In Section 5 we provide an axiomatic characterization of this class of share functions, using the "merger axiom". Section 6 concludes the paper.

\footnotetext{
${ }^{2}$ A value function is efficient if, for every TU-game, it exactly distributes the worth that can be obtained by the "grand coalition".

${ }^{3}$ The dummy axiom requires that a player who does not add to or detract from any coalition when he joins it earns a zero payoff. According to the additivity axiom, if we sum two TU-games then the payoff distributed in the sum game is equal to the sum of the payoff of separate games.
} 


\section{Games and values}

\subsection{TU-game}

A TU-game is a pair $(N, v)$ defined by a finite set of players $N$ and a function $v: 2^{N} \rightarrow \mathbb{R}$, that assigns to each coalition $S \subseteq N$ a real number $v(S)$ and satisfies $v(\varnothing)=0$. In this paper we consider monotone TU-games, i.e. games $(N, v)$ satisfying $v(S) \leq v(T)$ if $S \subseteq T \subseteq N$. We denote the collection of all monotone TU-games by $\mathcal{G}$. A monotone game is a null game if $v(S)=0$ for all $S \subseteq N$. Such a game on player set $N$ is denoted $\left(N, v^{0}\right)$, and $\mathcal{G}^{+}$ is the class of monotone games that are not null games. Henceforth when we refer to a game it will be a monotone game.

A value function on $\mathcal{G}$ is a function $\varphi$ that assigns to every $(N, v) \in \mathcal{G}$ an $|N|$-dimensional real vector $\varphi(N, v) \in$ $\mathbb{R}^{|N|}$. This vector can be seen as a distribution of payoffs over the individual players in the game.

Let $m_{S}^{i}(N, v)=v(S)-v(S \backslash\{i\})$ be the marginal contribution of player $i \in N$ to coalition $S \subseteq N$ in game $(N, v) \in \mathcal{G}$. The well-known Shapley value $\left(\varphi^{S h}\right)$ and Banzhaf value $\left(\bar{\varphi}^{B}\right)$ on the class $\mathcal{G}$ of monotone games are the functions defined by

$$
\varphi_{i}^{S h}(N, v)=\sum_{\substack{S \subseteq N \\ i \in S}} \frac{(|S|-1) !(|N|-|S|) !}{|N| !} m_{S}^{i}(N, v) \text { and } \bar{\varphi}_{i}^{B}(N, v)=\sum_{\substack{S \subseteq N \\ i \in S}} \frac{1}{2^{|N|-1}} m_{S}^{i}(N, v), i \in N .
$$

The Shapley value is characterized by the axioms of efficiency, null player, symmetry and additivity. The Banzhaf value satisfies the last three axioms, but not efficiency. So, to divide the worth $v(N)$ according to the Banzhaf value we replace the Banzhaf value by the normalized Banzhaf value $\left(\varphi^{B}\right)$, which is the efficient value function that distributes the worth $v(N)$ proportional to the Banzhaf values of the players, i.e.

$$
\varphi_{i}^{B}(N, v)=\frac{\bar{\varphi}_{i}^{B}(N, v)}{\sum_{i \in N} \bar{\varphi}_{i}^{B}(N, v)} v(N), i \in N,(N, v) \in \mathcal{G}^{+}, \text {and } \varphi_{i}^{B}\left(N, v^{0}\right)=0 \text { for all } i \in N .
$$

\subsection{Coalition structure}

A coalition structure on a player set $N$ is a finite partition $P_{0}=\left\{P_{1}, \ldots, P_{m}\right\}$ of $m$ non-empty, disjoint subsets of $N$, i.e. $\cup_{k=1}^{m} P_{k}=N$ and $P_{k} \cap P_{l}=\varnothing$ for all $k, l \in\{1, \ldots, m\}, k \neq l$. Note that a coalition structure is assumed to be given exogenously. In the following an element $P_{k}$ of the partition $P_{0}$ is called a structural coalition (Hamiache 1999). Since besides games between individual players, we will also consider games which set of players is formed by the elements in the coalition structure, we represent the set of structural coalitions in the coalition structure by the set $M=\{1, \ldots, m\}$, with $k \in M$ representing the structural coalition $P_{k} \in P$. Furthermore, the collection of all coalition structures is denoted by $\mathcal{P}_{0}^{N}$; a game with coalition structure $\left(N, v, P_{0}\right)$; and the collection of all games with coalition structure is denoted $\mathcal{G} \mathcal{P}_{0}^{N}$, with $\left(N, v, P_{0}\right) \in \mathcal{G} \mathcal{P}_{0}^{N}$.

A coalition structure value function $\theta$ (CS-value) on the set $\mathcal{G} \mathcal{P}_{0}^{N}$ assigns a payoff to any player in every game with coalition structure $\left(N, v, P_{0}\right)$. The arrangement of the players into a coalition structure implies two kinds of negotiation: among the structural coalitions of the coalition structure, and inside each of these structural coalitions. Owen (1977) generalized the Shapley value to games with coalition structure, and introduced two games to reflect such a two-level interaction structure.

For given $\left(N, v, P_{0}\right) \in \mathcal{G} \mathcal{P}_{0}^{N}$, with $P_{0}=\left\{P_{1}, \ldots, P_{m}\right\}$ and $M=\{1, \ldots, m\}$, the external game $e^{4}$ between structural coalitions $\left(M, v^{P}\right) \in \mathcal{G}$, is an $m$-player game defined by $v^{P}(L)=v(P(L))=v\left(\cup_{j \in L} P_{j}\right)$, for all $L \subseteq M$. In this game induced by $\left(N, v, P_{0}\right)$, when the structural coalitions of $P_{0}$ are considered as players the worth of the grand coalition is distributed amongst the structural coalitions. Observe that $v^{P}(M)=v(N)$.

The second game, the internal game $e^{5}$, takes place inside each structural coalition. In this game the payoff of each structural coalition is distributed amongst the players within this structural coalition. The internal game between the player in a structural coalition $P_{k}$, denoted $\left(P_{k}, v^{P_{k}}\right)$, is defined as a $\left|P_{k}\right|$-player game given by $v^{P_{k}}(S)=$ $\sum_{\substack{L \subset M \\ k \notin L}} \frac{|L| !(m-|L|-1) !}{m !} v^{P_{k}, L}(S), S \subseteq P_{k}$, where for $L \subset M, k \notin L, v^{P_{k}, L}(S)=v(S \cup P(L))-v(P(L))$, is the marginal contribution of $S \subseteq P_{k}$ to the union $P(L)$ of the structural coalitions $P_{j}, j \in L$. So, the internal game $\left(P_{k}, v^{P_{k}}\right)$ is a weighted sum of the games $\left(P_{k}, v^{P_{k}, L}\right)$, where the weight of the game $\left(P_{k}, v^{P_{k}, L}\right)$ is equal to the Shapley weight assigned to structural coalition $k \in M$ if this structural coalition joins the collection $L \subset M$ of structural coalitions.

\footnotetext{
${ }^{4}$ Owen (1977) introduced this game under the name of "quotient game".

${ }^{5}$ This definition (equivalent to those of Owen (1977)) is due to van den Brink and van der Laan (2005).
} 
The outcome of such a two-level interaction is reflected by the Owen-Shapley-CS value $\left(\theta^{O S}\right)$, which is for a player $i$ belonging to a structural coalition $P_{k}$ the Shapley value of this player in the internal game corresponding to $P_{k}$, that is

$$
\theta_{i}^{S h}\left(N, v, P_{0}\right)=\varphi_{i}^{S h}\left(P_{k}, v^{P_{k}}\right), i \in P_{k} \in P_{0}{ }^{6}
$$

Analogously, Owen (1981) generalized the Banzhaf value to games with coalition structure. In this case the outcome of the two-level interaction is reflected by the Owen-Banzhaf-CS value $\left(\bar{\theta}^{B}\right)$, which is for a player $i$ belonging to a structural coalition $P_{k}$ the Banzhaf (non normalized) value of this player in the internal game $\left(P_{k}, \bar{v}^{P_{k}}\right) \in \mathcal{G}$, corresponding to $P_{k}$. That is

$$
\bar{\theta}_{i}^{B}\left(N, v, P_{0}\right)=\bar{\varphi}_{i}^{B}\left(P_{k}, \bar{v}^{P_{k}}\right), i \in P_{k} \in P_{0}, \text { with } \bar{v}^{P_{k}}(S)=\sum_{\substack{L \subset M \\ k \notin L}} 2^{-(m-1)} v^{P_{k}, L}(S), S \subseteq P_{k} .
$$

In this case the internal game $\left(P_{k}, \bar{v}^{P_{k}}\right)$ is a weighted sum of the games $\left(P_{k}, v^{P_{k}, L}\right)$, where the weight of the game $\left(P_{k}, v^{P_{k}, L}\right)$ is equal to the Banzhaf weight assigned to structural coalition $k \in M$ if this structural coalition joins the collection $L \subset M$ of structural coalitions.

For axiomatizations of these two coalition structure values, we refer to Owen (1977), Hamiache (1999), Albizuri (2001) and Amer, Carreras and Giménez (2002), among others.

\subsection{Coalition configuration}

Games with coalition structure do not always reflect the complex relationships that may exist in a bargaining game. To solve this problem Albizuri et al. (2006a, 2006b) extended the approach of Owen $(1977,1981)$ in games where players are grouped in coalitions which are not necessarily disjoint.

A coalition configuration of $N$ is a finite collection $P=\left\{P_{1}, \ldots, P_{m}\right\}$, of $m$ non-empty subset of $N$, such that $\cup_{k=1}^{m} P_{k}=N$. The only assumption is that a player belongs to at least one coalition. An element $P_{r}$ of $P$ is called a configurational coalition. The set of configurational coalitions in the coalition configuration is also denoted $M=\{1, \ldots, m\}$. For every $i \in N$, we will write $P^{i}:\left\{P_{r} \in P: i \in P_{r}\right\}$ as the members of $P$, containing $i$. We denote by $\mathcal{P}^{N}$ and $\mathcal{G} \mathcal{P}^{N}$ the set of all coalition configurations and the set of all games with coalition configuration. $(N, v, P) \in \mathcal{G P}^{N}$ is a game with coalition configuration. Note that coalition structures are special cases of coalition configurations, when players can not belong to more than one configurational coalitions. This means that $\mathcal{G} \mathcal{P}_{0}^{N} \subseteq \mathcal{G} \mathcal{P}^{N}$.

A coalition configuration value function (CCF-value) $\eta$ on the set $\mathcal{G} \mathcal{P}^{N}$ assigns a payoff to any player in every game with coalition configuration $(N, v, P)$.

Albizuri et al. (2006a, 2006b) generalized the Shapley and the Banzhaf values to such games, as Owen (1977, 1981) generalized them to games with coalition structure. In order to do that, they also divided the bargaining process into two games, the internal and the external game. They introduced the two following values, the OwenShapley-CCF value $\left(\eta^{S h}\right)$ and the Owen-Banzhaf-CCF value $\left(\bar{\eta}^{B}\right)$, defined by

$$
\eta_{i}^{S h}(N, v, P)=\sum_{P_{r} \in P^{i}} \varphi_{i}^{S h}\left(P_{r}, v^{P_{r}}\right) \text { and } \bar{\eta}_{i}^{B}(N, v, P)=\sum_{P_{r} \in P^{i}} \bar{\varphi}_{i}^{B}\left(P_{r}, \bar{v}^{P_{r}}\right) \text {, for all } i \in N .
$$

The value received by a player $i$ in a game with coalition configuration must be equal to the sum, for each configurational coalition this player $i$ belongs to, of the Shapley value (Banzhaf value) of this player in the corresponding internal games. The main difference with CS-values is that a player can belong to more than one configurational coalition. We must therefore sum all the payoffs he receives in each configurational coalition to which he belongs. Note that, if $P$ is a partition, there is an empty intersection between two coalitions, then $\eta_{i}^{S h}(N, v, P)=\theta_{i}^{S h}(N, v, P)$ and $\bar{\eta}_{i}^{B}(N, v, P)=\bar{\theta}_{i}^{B}(N, v, P)$ for all $i \in N$.

Obviously Albizuri et al. (2006a, 2006b) characterized these two values. In particular, they proved that the Owen-Shapley-CCF value is efficient, whereas this is not the case of the Owen-Banzhaf-CCF value. Then, as suggested by van der Laan and van den Brink (1998), we will introduce share functions in order to solve this problem. Before presenting share functions for games with coalition configuration, let us summarize the main results of share functions in the following section.

\footnotetext{
${ }^{6}$ In this formulation, the external game does not appear clearly, but is useful to determine the payoff in the internal game.
} 


\section{Share function}

\subsection{Share functions for TU-games}

A share function assigns to each player in a game $(N, v)$ its share in the worth of the grand coalition. More formally, a share function on $\mathcal{G}$ is a function $\rho$ which assigns to every game $(N, v) \in \mathcal{G}$ exactly one share vector, $\rho(N, v) \in \mathbb{R}^{|N|}$, such that $\sum_{i \in N} \rho_{i}(N, v)=1$.

The Shapley share function $\rho^{S h}$ on $\mathcal{G}$ is defined by

$$
\rho_{i}^{S h}(N, v)=\frac{\varphi_{i}^{S h}(N, v)}{v(N)}, i \in N,(N, v) \in \mathcal{G}^{+} \text {and } \rho_{i}^{S h}(N, v)=\frac{1}{|N|}, i \in N \text {, when } v=v^{0} .
$$

The Banzhaf share function $\rho^{B}$ on $\mathcal{G}$ is defined by

$$
\rho^{B}(N, v)=\frac{\bar{\varphi}_{i}^{B}(N, v)}{\sum_{i \in N} \bar{\varphi}_{i}^{B}(N, v)}=\frac{\varphi_{i}^{B}(N, v)}{v(N)}, i \in N,(N, v) \in \mathcal{G}^{+} \text {and } \rho_{i}^{B}(N, v)=\frac{1}{|N|}, i \in N \text {, when } v=v^{0} .
$$

Next we state some properties for a real valued function $\mu: \mathcal{G} \rightarrow \mathbb{R}$. Let $\mathcal{C} \subseteq \mathcal{G}$. A function $\mu: \mathcal{C} \rightarrow \mathbb{R}$ is called positive on $\mathcal{C} \subseteq \mathcal{G}$ if $\mu\left(N, v^{0}\right)=0$ and $\mu(N, v)>0$ for every $(N, v) \in \mathcal{C} \cap \mathcal{G}^{+}$. It is called additive on $\mathcal{C}$ if for every pair $(N, v)$ and $(N, w) \in \mathcal{C}$ satisfying $(N, v+w) \in \mathcal{C}$, it holds that $\mu(N, v+w)=\mu(N, v)+\mu(N, w)$. And finally, this function is symmetric on $\mathcal{C}$ if for every $(N, v) \in \mathcal{C}$, every pair of symmetric players ${ }^{7} i$ and $j$ in $(N, v)$, and every $S \subseteq N$ such that $\{i, j\} \subseteq S$ and both restricted games $\left(S \backslash\{i\}, v_{S \backslash\{i\}}\right)$ and $\left(S \backslash\{j\}, v_{S \backslash\{j\}}\right)$ are in $\mathcal{C}$, it holds that $\mu\left(S \backslash\{i\}, v_{S \backslash\{i\}}\right)=\mu\left(S \backslash\{j\}, v_{S \backslash\{j\}}\right)$.

Van der Laan and van den Brink (1998) defined a general share functions for TU-games which contains, among others, the Shapley share and the Banzhaf share functions. For given positive vectors $w^{|N|} \in \mathbb{R}_{+}^{|N|},|N| \in \mathbb{N}$, with $w_{i}^{|N|}>0$ for all $i \in\{1, \ldots,|N|\}$, let the function $\mu^{w}: \mathcal{G}^{+} \rightarrow \mathbb{R}$ be defined by $\mu^{w}(N, v)=\sum_{i \in N} \sum_{\substack{S \subseteq N \\ i \in N}} w_{|S|}^{|N|} m_{s}^{i}(N, v)$. Then the share function $\rho^{\mu^{w}}(N, v)$ on $\mathcal{G}^{+}$is given by

$$
\rho_{i}^{\mu^{w}}(N, v)=\frac{\sum_{\substack{S \subseteq N \\ i \in N}} w_{|S|}^{|N|} m_{S}^{i}(N, v)}{\mu^{w}(N, v)} \text { for all } i \in N \text { and }(N, v) \in \mathcal{G}^{+} \text {. }
$$

We have a general formula for the share functions where the two variables $\mu^{w}(N, v)$ and $w_{|S|}^{|N|}$ can be interpreted as the sum of payoffs of all the players in the game $(N, v)$, and as a weighted system. Note that for any system of positive weights the corresponding function $\mu^{w}$ is positive, symmetric and additive on $\mathcal{G}^{+}$.

In van der Laan and van den Brink (1998) it is shown that, when $\mu^{w}=\mu^{S h}=v(N)$, we have the Shapley share function and, when $\mu^{w}=\mu^{B}=\sum_{S \subseteq N}(2|S|-|N|) v(S)$, we have the Banzhaf share function. They also introduced the Deegan-Packel share $\rho_{i}^{\mu^{D P}}(N, v)$, which belongs to this class of share functions ${ }^{8}$.

Note that the share functions $\rho^{\mu^{w}}$ are defined on the class $\mathcal{G}^{+}$of monotone non-null games. Therefore we can extend the concept of share functions to null games by giving all players an equal share ${ }^{9}$, i.e.

$$
\rho^{\mu^{w}}\left(N, v^{0}\right)=\frac{1}{|N|} \text { for all } i \in N .
$$

\subsection{Share functions for games with coalition structure}

Owen (1977) asserted that all values defined for games with coalition structure should satisfy the property of "multiplication". According to this property, the fraction in the total payment $v(N)$ received by a player $i$ belonging to a structural coalition $P_{k}$ should be equal to the product of the fraction that the structural coalition $P_{k}$ receives in the external game between structural coalitions (structural coalitions split $v(M)$ ), and the fraction that this player $i$ receives in the internal game, when the same value function is applied to these two games. Implicitly Owen (1977) stated this property, using share functions. Van der Laan and van den Brink (2002) and, van den Brink and van der Laan (2005) thus applied this to the Owen-Shapley-CS value and the Owen-Banzhaf-CS value in order to obtain share functions for games with coalition structure.

\footnotetext{
$7_{i}$ and $j$ are symmetric in $(N, v) \in \mathcal{G}$ if $v(S \cup\{i\})=v(S \cup\{j\})$ for all $S \subseteq N \backslash\{i, j\}$.

${ }^{8}$ For a clear definition, see van der Laan and van den Brink (1998) and Deegan-Packel (1979).

${ }^{9}$ See van den Brink and van der Laan (2005) for a discussion about null games and share functions.
} 
A coalition structure share function (CS-share) is a function $\psi$ on $\mathcal{G P}_{0}^{N}$ satisfying $\sum_{i \in N} \psi_{i}\left(N, v, P_{0}\right)=1$ for all $\left(N, v, P_{0}\right) \in \mathcal{G} \mathcal{P}_{0}^{N}$ and assigns to every player in a game with coalition structure a share in the worth to be distributed.

The Owen-Shapley-CS share function for games with coalition structure $\left(\psi^{S h}\right)$ assigns to every player in a game with coalition structure its share according to the Owen-Shapley-CS value. $\psi_{i}^{S h}\left(N, v, P_{0}\right)$ is obtained by dividing the Owen-Shapley-CS value by $v(N)$ :

$$
\psi_{i}^{S h}\left(N, v, P_{0}\right)=\frac{\theta_{i}^{S h}\left(N, v, P_{0}\right)}{v(N)}, i \in N .
$$

Applying the multiplication property, the Owen-Shapley-CS share of player $i \in P_{k} \in P_{0}$, can be reformulated as the product of two Shapley shares: the first share is the share $\rho_{k}^{S h}\left(M, v^{P}\right)$ of coalition $k$ in the external game $\left(M, v^{P}\right)$ between structural coalitions; and the second share is the share $\rho_{i}^{S h}\left(P_{k}, v^{P_{k}}\right)$ of player $i$ in the internal game $\left(P_{k}, v^{P_{k}}\right)$.

Then for given $\left(N, v, P_{0}\right) \in \mathcal{G} \mathcal{P}_{0}^{N}$ with $P_{0}=\left\{P_{1}, \ldots, P_{m}\right\}$ and $M=\{1, \ldots, m\}$, van der Laan and van den Brink (2002) and, van den Brink and van der Laan (2005) showed that:

$$
\psi_{i}^{S h}\left(N, v, P_{0}\right)=\rho_{i}^{S h}\left(P_{k}, v^{P_{k}}\right) \cdot \rho_{k}^{S h}\left(M, v^{P}\right), i \in P_{k}, k \in M, \text { with } v^{P_{k}}(S)=\sum_{\substack{L \notin M \\ k \notin L}} \frac{|L| !(m-|L|-1) !}{m !} v^{P_{k}, L}(S), S \subseteq P_{k} .
$$

Analogously the Banzhaf-CS share function $\left(\psi^{B}\right)$ assigns to every player $i$ belonging to a structural coalition $P_{k}$, the product of the Banzhaf share of coalition $P_{k}$ in the external game between structural coalitions, and the Banzhaf share of player $i$ in the internal game corresponding to $P_{k}$.

$$
\psi_{i}^{B}\left(N, v, P_{0}\right)=\rho_{i}^{B}\left(P_{k}, \bar{v}^{P_{k}}\right) \cdot \rho_{k}^{B}\left(M, v^{P}\right), i \in P_{k}, k \in M, \text { with } \bar{v}^{P_{k}}=\sum_{\substack{L \subset M \\ k \notin L}} 2^{-(m-1)} v^{P_{k}, L}(S), S \subseteq P_{k} .
$$

As already proved by van der Laan and van den Brink (2002) and van den Brink and van der Laan (2005), in general, the share of a player $i$ according to the Banzhaf-CS share function is different from the share function that is obtained from the Owen-Banzhaf-CS value (named Owen-Banzhaf-CS share). Indeed, by definition, the Banzhaf-CS share function satisfies the multiplication property, which is not the case for the Owen-Banzhaf-CS share function.

\subsection{A class of share functions for games with coalition structure}

We present now a class of share functions for games with coalition structure. The class of share functions considered here is close to the class considered in van den Brink and van der Laan (2005), although they are different. A discussion of this difference is given at the end of this part.

As argued before, for games with coalition structure the payoff of a player can be seen as the result of a first level game between coalitions and a second level game between the players within a coalition. Let $\mu: \mathcal{G} \rightarrow \mathbb{R}$ be an additive, positive and symmetric function on $\mathcal{G}$, game $(N, v) \in \mathcal{G}$ and a coalition structure $P_{0} \in \mathcal{P}_{0}^{N}$, then we have the two following games.

The first one is the external game $\left(M, v^{P}\right) \in \mathcal{G}$ introduced previously to define the Owen-Shapley-CS value and the Owen-Banzhaf-CS value. The second one is the $\mu$-internal game which is a generalization of $v^{P_{k}}(S)$ and $\bar{v}^{P_{k}}(S)$. For $P_{k} \in P_{0}$, we define the $\mu$-internal game $\left(P_{k}, v_{\mu}^{P_{k}}\right)$ by $v_{\mu}^{P_{k}}(S)=\rho_{k}^{\mu}\left(M, v_{P_{k} \mid S}^{P}\right) \cdot \mu\left(M, v_{P_{k} \mid S}^{P}\right), S \subseteq P_{k}$, where $M=\{1, \ldots, m\}, \mu\left(M, v_{P_{k} \mid S}^{P}\right)$ positive and the game $\left(M, v_{P_{k} \mid S}^{P}\right)$ on the player set $M$ of structural coalitions is given by the characteristic function $v_{P_{k} \mid S}^{P}, S \subseteq P_{k} \in P_{0}$, defined by $v_{P_{k} \mid S}^{P}(L)=\left\{\begin{array}{c}v(P(L \backslash\{k\}) \cup S) \text { if } k \in L \subseteq M \\ v(P(L)) \text { if } k \notin L \subset M\end{array} \mid\right.$. So $v_{P_{k} \mid S}^{P}$ assigns to every coalition $L$ (of structural coalitions in the coalition structure) the worth of the union of these coalitions, where structural coalition $P_{k}$ is replaced by $S \subseteq P_{k}$. That is, player $l \in M \backslash\{k\}$ represents an a priori structural coalition $P_{l} \neq P_{k}$ in the coalition structure $P_{0}$, whereas player $k$ represents the sub-coalition $S \subseteq P_{k}$ instead of $P_{k}$ as a whole. Notice that this game is named by Owen (1977) as the "almost quotient game".

We introduce in Proposition 1 a class of share functions for games with coalition structure using the multiplication property. 
Proposition 1. Let $\mu: \mathcal{G} \rightarrow \mathbb{R}$ be an additive, positive and symmetric function on $\mathcal{G}$ and let $\rho^{\mu}$ a share function as defined in Subsection 3.1. Then the function $\psi^{\mu}$ on $\mathcal{G P}_{0}^{N}$ defined by

$$
\psi_{i}^{\mu}\left(N, v, P_{0}\right)=\rho_{i}^{\mu}\left(P_{k}, v_{\mu}^{P_{k}}\right) \cdot \rho_{k}^{\mu}\left(M, v^{P}\right),
$$

for $i \in P_{k} \in P_{0}=\left\{P_{1}, \ldots, P_{m}\right\} \in \mathcal{P}_{0}^{N}, k \in M$, is a CS-share function and satisfies for every $\left(N, v, P_{0}\right) \in \mathcal{G} \mathcal{P}_{0}^{N}$ the following properties:

i) $\sum_{i \in P_{k}} \psi_{i}^{\mu}\left(N, v, P_{0}\right)=\rho_{k}^{\mu}\left(M, v^{P}\right)$

ii) $\psi_{i}^{\mu}\left(N, v, P_{0}\right)=\rho_{i}^{\mu}(N, v)$ when $P_{0}=\{N\}$,

iii) $\psi_{i}^{\mu}\left(N, v, P_{0}\right)=\rho_{i}^{\mu}(N, v)$ when $P_{0}=\{\{i\}, i \in N\}$.

Proof. See the similar proof of Theorem 3.1 in van den Brink and van der Laan (2005).

According to the first property this function is consistent, in the sense that the sum of the payoffs of all the players in a structural coalition $P_{k}$ is equal to the payoff received by this structural coalition in the external game. The second and third properties show that $\psi^{\mu}$ generalizes $\rho^{\mu}$, because when there is only one structural coalition in the coalition structure, or when there are $n$ singleton structural coalitions in the coalition structure, the class of share functions for games with coalition structure corresponds to the class of share functions for TU-games.

Proposition 2 shows how to obtain the Owen-Shapley-CS share function and the Banzhaf-CS share function from $\psi^{\mu}$.

Proposition 2. The CS-share function for games with coalition structure $\psi^{\mu}$ is

i) Owen-Shapley-CS share function $\psi^{S h}$ when $\mu(N, v)=\mu^{S h}(N, v)=v(N)$,

ii) Banzhaf-CS share function $\psi^{B}$ when $\mu(N, v)=\mu^{B}(N, v)=\frac{1}{2^{|N|-1}} \sum_{S \subseteq N}(2|S|-|N|) v(S)$.

Proof. See the similar proof of Proposition 3.2 in van den Brink and van der Laan (2005).

From this formula, we can also obtain other share functions for games with coalition structure. Especially, we can obtain a Deegan-Packel-CS share function. This function denoted $\psi_{i}^{\mu^{D P}}\left(N, v, P_{0}\right)$ is given by

$$
\psi_{i}^{\mu^{D P}}\left(N, v, P_{0}\right)=\rho_{i}^{\mu^{D P}}\left(P_{k}, v_{\mu^{D P}}^{P_{k}}\right) \cdot \rho_{k}^{\mu^{D P}}\left(M, v^{P}\right), i \in N .
$$

Our class of shares functions is different from the class defined in van den Brink and van der Laan (2005), because the internal game defined in this paper does not in general coincide with the internal game defined by these authors. Indeed, both games coincide if they are the ones associated with the Owen-Shapley-CS share function or the Deegan-Packel-CS share function. But the Banzhaf-CS share function is not included in the class considered by van den Brink and van der Laan (2005), since the second part in Proposition 3.2 by van den Brink and van der Laan (2005) is not true. Indeed, in their paper, mistakenly $\varphi_{k}^{B}\left(M, v_{P_{k} \mid S}^{P}\right)$ is supposed to be equal to $\bar{v}^{P_{k}}(S)$ instead of $\bar{\varphi}_{k}^{B}\left(M, v_{P_{k} \mid S}^{P}\right)$, and therefore $v_{\mu}^{P_{k}}$ should be defined with respect to the $\mu$ function instead of the $v$ function as they did in their paper.

To conclude this section, note that van den Brink and van der Laan (2005) proposed different characterizations of this class of share functions for games with coalition structure. While their families are slightly different from ours, their axioms also characterize our class of share functions. Indeed we have the same set of axioms, but within the axioms the games $v_{\mu}^{P_{k}}$ are different. We do not present them here, but most of these axioms will be useful in characterizing the class of share functions for games with coalition configuration, that we will present now.

\section{Share functions for games with coalition configuration}

\subsection{Owen-Shapley-CCF share and Banzhaf-CCF share}

Let us begin with the definition of a share function for games with coalition configuration (CCF-share). This function $\Pi$ on $\mathcal{G} \mathcal{P}^{N}$ assigns to every player in a game with coalition configuration a share in the worth to be distributed, such that $\sum_{i \in N} \Pi_{i}(N, v, P)=1$ for all $(N, v, P) \in \mathcal{G} \mathcal{P}^{N}$.

Therefore the Owen-Shapley-CCF share function, which assigns to every player in a game with coalition configuration its share according to the Owen-Shapley-CCF value, is the function $\Pi_{i}^{S h}(N, v, P)$ given by

$$
\Pi_{i}^{S h}(N, v, P)=\frac{\eta_{i}^{S h}(N, v, P)}{\sum_{i \in N} \eta_{i}^{S h}(N, v, P)}=\frac{\eta_{i}^{S h}(N, v, P)}{v(N)}, i \in N
$$


with $\eta_{i}^{S h}(N, v, P)=\sum_{P r \in P^{i}} \varphi_{i}^{S h}\left(P_{r}, v^{P_{r}}\right)$.

The Owen-Shapley-CCF share function of a player $i$ can also be reformulated, as the sum for each configurational coalition this player $i$ belongs to, as the product of two Shapley shares:

- the first share is the share $\rho_{r}^{S h}\left(M, v^{P}\right)$ of coalitions $r$ in the external game $\left(M, v^{P}\right)$ between configurational coalitions;

- and the second share is the share $\rho_{i}^{S h}\left(P_{r}, v^{P_{r}}\right)$ of player $i$ in the internal games $\left(P_{r}, v^{P_{r}}\right)$ corresponding to $P_{r}$.

The external game and internal game are identical to those introduced to characterize the Owen-Shapley-CS share function.

We then obtain the following proposition.

Proposition 3. Let $(N, v, P)$ be a game on $\mathcal{G P}^{N}, P=\left\{P_{1}, \ldots, P_{m}\right\}$ a coalition configuration of $N$ and $i \in N$, then the Owen-Shapley-CCF share function of a player $i$ is given by

$$
\Pi_{i}^{S h}(N, v, P)=\sum_{P_{r} \in P^{i}}\left[\rho_{i}^{S h}\left(P_{r}, v^{P_{r}}\right) \cdot \rho_{r}^{S h}\left(M, v^{P}\right)\right],
$$

with $v^{P_{r}}(S)=\sum_{\substack{L \subset M \\ r \notin L}} \frac{|L| !(m-|L|-1) !}{m !} v^{P_{r}, L}(S)$.

Proof. Since $\rho_{i}^{S h}\left(P_{r}, v^{P_{r}}\right)=\frac{\varphi_{i}^{S h}\left(P_{r}, v^{P_{r}}\right)}{v^{P_{r}}\left(P_{r}\right)}$, then $\varphi_{i}^{S h}\left(P_{r}, v^{P_{r}}\right)=\rho_{i}^{S h}\left(P_{r}, v^{P_{r}}\right) \cdot v^{P_{r}}\left(P_{r}\right)$. Therefore $\Pi_{i}^{S h}(N, v, P)=$ $\frac{\eta_{i}^{S h}(N, v, P)}{v(N)}=\frac{\sum_{P_{r} \in P^{i}} \varphi_{i}^{S h}\left(P_{r}, v^{P_{r}}\right)}{v(N)}=\frac{\sum_{P_{r} \in P^{i}}\left[\rho_{i}^{S h}\left(P_{r}, v^{P_{r}}\right) \cdot v^{P_{r}}\left(P_{r}\right)\right]}{v(N)}$. Since $v(N)$ is given, then $\frac{\sum_{P_{r} \in P^{i}}\left[\rho_{i}^{S h}\left(P_{r}, v^{P_{r}}\right) \cdot v^{P_{r}}\left(P_{r}\right)\right]}{v(N)}$ $=\sum_{P_{r} \in P^{i}}\left[\rho_{i}^{S h}\left(P_{r}, v^{P_{r}}\right) \cdot \frac{v^{P_{r}}\left(P_{r}\right)}{v(N)}\right]$. And we know that $v^{P_{r}}\left(P_{r}\right)=\varphi_{r}^{S h}\left(M, v^{P}\right)$ and $v(N)=v^{P}(M)$ (van den Brink and van der Laan (2005)). This means that $\sum_{P_{r} \in P^{i}}\left[\rho_{i}^{S h}\left(P_{r}, v^{P_{r}}\right) \cdot \frac{v^{P_{r}}\left(P_{r}\right)}{v(N)}\right]=\sum_{P_{r} \in P^{i}}\left[\rho_{i}^{S h}\left(P_{r}, v^{P_{r}}\right) \cdot \frac{\varphi_{r}^{S h}\left(M, v^{P}\right)}{v^{P}(M)}\right]$. By definition $\rho_{r}^{S h}\left(M, v^{P}\right)=\frac{\varphi_{r}^{S h}\left(M, v^{P}\right)}{v(N)}$, then $\Pi_{i}^{S h}(N, v, P)=\sum_{P_{r} \in P^{i}}\left[\rho_{i}^{S h}\left(P_{r}, v^{P_{r}}\right) . \rho_{r}^{S h}\left(M, v^{P}\right)\right]$.

The main difference with respect to the Owen-Shapley-CS share function is that, since a player can belong to more than one configurational coalition, we must sum all the payoffs he receives in each of the configurational coalitions he belongs to.

Applying a similar argument, we can now define the Banzhaf-CCF share function. However the Owen-Banzhaf$\mathrm{CCF}$ value is not efficient (see section 2), and so does not satisfy the multiplication property. Consequently, we must first define a $C C F$-value, different from $\bar{\eta}_{i}^{B}(N, v, P)$, which verifies the multiplication property. This is the Banzhaf-CCF value, denoted $\eta_{i}^{B}(N, v, P)$ and given by

$$
\eta_{i}^{B}(N, v, P)=\sum_{P_{r} \in P^{i}} \varphi_{i}^{B}\left(P_{r}, \bar{v}^{P_{r}}\right), \text { for all } i \in N .
$$

This value is, for a player $i$, the sum for all configurational coalitions he belongs to of the normalized Banzhaf value of this player in the corresponding internal games $\left(P_{r}, \bar{v}^{P_{r}}\right) \in \mathcal{G}$.

Hence, the Banzhaf-CCF share function which assigns to every player in a game with coalition configuration its share according to the Banzhaf-CCF value is the function denoted $\Pi_{i}^{B}(N, v, P)$ and given by

$$
\Pi_{i}^{B}(N, v, P)=\frac{\eta_{i}^{B}(N, v, P)}{\sum_{i \in N} \eta_{i}^{B}(N, v, P)}, i \in N
$$

with $\eta_{i}^{B}(N, v, P)=\sum_{P_{r} \in P^{i}} \varphi_{i}^{B}\left(P_{r}, \bar{v}^{P_{r}}\right)$.

The Banzhaf-CCF share function of player $i$ is also, for each configurational coalition $i$ belongs to, the result of the product of two Banzhaf shares: the Banzhaf share of $i$ in the corresponding internal game $\left(P_{r}, \bar{v}^{P_{r}}\right)$ and the Banzhaf share of configurational coalitions $r$ in the external game $\left(M, v^{P}\right)$.

Proposition 4. Let $(N, v, P)$ be a game on $\mathcal{G P}^{N}, P=\left\{P_{1}, \ldots, P_{m}\right\}$ a coalition configuration of $N$ and $i \in N$, then the Banzhaf-CCF share function of a player $i$ is given by

$$
\Pi_{i}^{B}(N, v, P)=\sum_{P_{r} \in P^{i}}\left[\rho_{i}^{B}\left(P_{r}, \bar{v}^{P_{r}}\right) \cdot \rho_{r}^{B}\left(M, v^{P}\right)\right]
$$

with $\bar{v}^{P_{r}}=\sum_{\substack{L \subset M \\ k \notin L}} 2^{-(m-1)} v^{P, L}(S)$. 
Proof. We proceed in three steps:

1) To begin, we prove that $\sum_{i \in N} \eta_{i}^{B}(N, v, P)=\sum_{r \in M} \bar{\varphi}_{r}^{B}\left(M, v^{P}\right)$. By definition $\eta_{i}^{B}(N, v, P)=\sum_{P_{r} \in P^{i}} \varphi_{i}^{B}\left(P_{r}, \bar{v}^{P_{r}}\right)$, then $\sum_{i \in N} \eta_{i}^{B}(N, v, P)=\sum_{i \in N} \sum_{P_{r} \in P^{i}} \varphi_{i}^{B}\left(P_{r}, \bar{v}^{P_{r}}\right)=\sum_{r \in M} \sum_{i \in P_{r}} \sum_{P_{r} \in P^{i}} \varphi_{i}^{B}\left(P_{r}, \bar{v}^{P_{r}}\right)=\sum_{r \in M} \sum_{i \in P_{r}} \varphi_{i}^{B}\left(P_{r}, \bar{v}^{P_{r}}\right)$ for all $r \in M$. And since $\varphi_{i}^{B}\left(P_{r}, \bar{v}^{P_{r}}\right)=\frac{\bar{\varphi}_{i}^{B}\left(P_{r}, \bar{v}^{P_{r}}\right) .}{\sum_{i \in P_{r}} \bar{\varphi}_{i}^{B}\left(P_{r}, \bar{v}^{P_{r}}\right)} \bar{v}^{P_{r}}\left(P_{r}\right)$, then $\sum_{i \in N} \eta_{i}^{B}(N, v, P)$

$=\sum_{r \in M} \sum_{i \in P_{r}}\left[\frac{\bar{\varphi}_{i}^{B}\left(P_{r}, \bar{v}_{r}\right) .}{\sum_{i \in P_{r}} \bar{\varphi}_{i}^{B}\left(P_{r}, \bar{v}^{P_{r}}\right)} \bar{v}^{P_{r}}\left(P_{r}\right)\right]=\sum_{r \in M} \bar{v}^{P_{r}}\left(P_{r}\right)\left[\frac{\sum_{i \in P_{r}} \bar{\varphi}_{i}^{B}\left(P_{r}, \bar{v}^{P_{r}}\right)}{\sum_{i \in P_{r}} \bar{\varphi}_{i}^{B}\left(P_{r}, \bar{v}^{P_{r}}\right)}\right]=\sum_{r \in M} \bar{v}^{P_{r}}\left(P_{r}\right)$. And since $\bar{v}^{P_{r}}\left(P_{r}\right)=\bar{\varphi}_{r}^{B}\left(M, v^{P}\right)$,

then $\sum_{i \in N} \eta_{i}^{B}(N, v, P)=\sum_{r \in M} \bar{\varphi}_{r}^{B}\left(M, v^{P}\right)$ (a).

2) We provide now that $\eta_{i}^{B}(N, v, P)=\sum_{P_{r} \in P^{i}}\left[\rho_{i}^{B}\left(P_{r}, \bar{v}^{P_{r}}\right) \cdot \bar{\varphi}_{r}^{B}\left(M, v^{P}\right)\right]$. By definition $\rho_{i}^{B}\left(P_{r}, \bar{v}^{P_{r}}\right)=\frac{\varphi_{i}^{B}\left(P_{r}, \bar{v}^{P_{r}}\right)}{\bar{v}_{r}\left(P_{r}\right)}$ then $\varphi_{i}^{B}\left(P_{r}, \bar{v}^{P_{r}}\right)=\rho_{i}^{B}\left(P_{r}, \bar{v}^{P_{r}}\right) \cdot \bar{v}^{P_{r}}\left(P_{r}\right)$. Therefore $\eta_{i}^{B}(N, v, P)=\sum_{P_{r} \in P^{i}} \varphi_{i}^{B}\left(P_{r}, \bar{v}^{P_{r}}\right)=\sum_{P_{r} \in P^{i}}\left[\rho_{i}^{B}\left(P_{r}, \bar{v}^{P_{r}}\right) \cdot \bar{v}^{P_{r}}\left(P_{r}\right)\right]$, then $\eta_{i}^{B}(N, v, P)=\sum_{P_{r} \in P^{i}}\left[\rho_{i}^{B}\left(P_{r}, \bar{v}^{P_{r}}\right) \cdot \bar{\varphi}_{r}^{B}\left(M, v^{P}\right)\right](\mathrm{b})$.

3) Finally we show that $\Pi_{i}^{B}(N, v, P)=\sum_{P r \in P^{i}}\left[\rho_{i}^{B}\left(P_{r}, \bar{v}^{P_{r}}\right) \cdot \rho_{r}^{B}\left(M, v^{P}\right)\right]$. By definition $\Pi_{i}^{B}(N, v, P)=\frac{\eta_{i}^{B}(N, v, P)}{\sum_{i \in N} \eta_{i}^{B}(N, v, P)}$, and from (a) and (b), $\Pi_{i}^{B}(N, v, P)=\frac{\sum_{P_{r} \in P^{i}}\left[\rho_{i}^{B}\left(P_{r}, \bar{v}^{P_{r}}\right) \cdot \bar{\varphi}_{r}^{B}\left(M, v^{P}\right)\right]}{\sum_{r \in M} \bar{\varphi}_{r}^{B}\left(M, v^{P}\right)}=\sum_{P_{r} \in P^{i}} \frac{\left[\rho_{i}^{B}\left(P_{r}, \bar{v}^{P_{r}}\right) \cdot \bar{\varphi}_{r}^{B}\left(M, v^{P}\right)\right]}{\sum_{r \in M} \bar{\varphi}_{r}^{B}\left(M, v^{P}\right)}$ $=\sum_{P_{r} \in P^{i}}\left[\rho_{i}^{B}\left(P_{r}, \bar{v}^{P_{r}}\right) \cdot \frac{\bar{\varphi}_{r}^{B}\left(M, v^{P}\right)}{\sum_{r \in M} \bar{\varphi}_{r}^{B}\left(M, v^{P}\right)}\right]=\sum_{P_{r} \in P^{i}}\left[\rho_{i}^{B}\left(P_{r}, \bar{v}^{P_{r}}\right) \cdot \rho_{r}^{B}\left(M, v^{P}\right)\right]$.

For the same reason as the Banzhaf-CS share function is different from the CS-share function that is obtained by normalizing the Owen-Banzhaf-CS value, the Banzhaf-CCF share function $\Pi_{i}^{B}(N, v, P)$ is different in general from $\bar{\Pi}_{i}^{B}(N, v, P)=\frac{\bar{\eta}_{i}^{B}(N, v, P)}{\sum_{i \in N} \bar{\eta}_{i}^{B}(N, v, P)}$, which is obtained by normalizing the Owen-Banzhaf-CCF value.

\subsection{A class of share functions for games with coalition configuration}

We now generalize the share function $\rho^{\mu}$ to games with coalition configuration. As already noted, for such a game the payoff of a player can be seen as the sum for each configurational coalition this player belongs to, as the result of a first-level game between configurational coalitions and a second-level game between the players within a configurational coalition. The first game is the external game $\left(M, v^{P}\right)$, and the second game the $\mu$-internal game $\left(P_{r}, v_{\mu}^{P_{r}}\right)$.

Proposition 5. Let $\mu: \mathcal{G} \rightarrow \mathbb{R}$ be an additive, positive and symmetric function on $\mathcal{G}$, and let $\rho^{\mu}$ a share function for $T U$-game as defined in Subsection 3.1. Then the function $\Pi^{\mu}$ on $\mathcal{G P}^{N}$ given by

$$
\Pi_{i}^{\mu}(N, v, P)=\sum_{P_{r} \in P^{i}}\left[\rho_{i}^{\mu}\left(P_{r}, v_{\mu}^{P_{r}}\right) \cdot \rho_{r}^{\mu}\left(M, v^{P}\right)\right]
$$

for all $i \in N, P^{i}:\left\{P_{r} \in P: i \in P_{r}\right\}$, is a $C C F$-share function and satisfies for every $(N, v, P) \in \mathcal{G P}^{\mathcal{N}}$ the following properties:

i) $\sum_{i \in P_{k}} \Pi_{i}^{\mu}(N, v, P)=\sum_{i \in P_{k}} \sum_{P_{r} \in P^{i}}\left[\rho_{i}^{\mu}\left(P_{r}, v_{\mu}^{P_{r}}\right) \cdot \rho_{r}^{\mu}\left(M, v^{P}\right)\right]$,

ii) $\Pi_{i}^{\mu}(N, v, P)=\psi_{i}^{\mu}(N, v, P)=\rho_{i}^{\mu}(N, v)$ when $P=\{N\}$,

iii) $\Pi_{i}^{\mu}(N, v, P)=\psi_{i}^{\mu}(N, v, P)=\rho_{i}^{\mu}(N, v)$ when $P=\{\{i\}, i \in N\}$.

Proof. Since $\rho^{\mu}$ is a share function, then $\sum_{i \in N} \rho_{i}^{\mu}(N, v)=1$, and $\sum_{i \in N} \Pi_{i}^{\mu}(N, v, P)=\sum_{k \in M} \sum_{i \in P_{k}} \Pi_{i}^{\mu}(N, v, P)$ $=\sum_{k \in M} \sum_{i \in P_{k}}\left(\sum_{P_{r} \in P^{i}}\left[\rho_{i}^{\mu}\left(P_{r}, v_{\mu}^{P_{r}}\right) \cdot \rho_{r}^{\mu}\left(M, v^{P}\right)\right]\right)=$

$\left(\sum_{k \in M} \sum_{P_{r} \in P^{i}} \rho_{r}^{\mu}\left(M, v^{P}\right)\right) \cdot\left(\sum_{i \in P_{k}} \rho_{i}^{\mu}\left(P_{r}, v_{\mu}^{P_{r}}\right)\right)$. And since we apply $\sum_{i \in P_{k}}$ to all the $P_{k}$ in $M$, we apply it also to all the $P_{r} \in P^{i}$. Likewise, since we apply $\sum_{k \in M}$ to all the $P_{k}$ in $M$, we apply it also to all the $P_{r} \in P^{i}$. Then $\sum_{i \in N} \Pi_{i}^{\mu}(N, v, P)=\left(\sum_{k \in M} \rho_{k}^{\mu}\left(M, v^{P}\right)\right) \cdot\left(\sum_{i \in P_{r}} \rho_{i}^{\mu}\left(P_{r}, v_{\mu}^{P_{r}}\right)\right)=1$. Therefore $\Pi^{\mu}(N, v, P)$ is a share function.

Moreover we have

i) Straightforward by definition.

ii) Since $P=\left\{P_{1}\right\}$ with $P_{1}=N$ and $M=\{1\}$ then $P^{i}=P_{r}$. Then $\Pi_{i}^{\mu}(N, v, P)=\sum_{P_{r} \in P^{i}}\left[\rho_{i}^{\mu}\left(P_{r}, v_{\mu}^{P_{r}}\right) . \rho_{r}^{\mu}\left(M, v^{P}\right)\right]$ $=\left[\rho_{i}^{\mu}\left(P_{r}, v_{\mu}^{P_{r}}\right) \cdot \rho_{r}^{\mu}\left(M, v^{P}\right)\right]=\psi_{i}^{\mu}(N, v, P)$.

And by $i i)$ of proposition 1 we know that $\psi_{i}^{\mu}(N, v, P)=\rho_{i}^{\mu}(N, v)$. Then $\Pi_{i}^{\mu}(N, v, P)=\psi_{i}^{\mu}(N, v, P)=\rho_{i}^{\mu}(N, v)$.

iii) Since $P=\left\{P_{1}\right\}$ with $P_{1}=\{i\}$ and $M=\{1\}$ then $P^{i}=P_{r}$. Then $\Pi_{i}^{\mu}(N, v, P)=\sum_{P_{r} \in P^{i}}\left[\rho_{i}^{\mu}\left(P_{r}, v_{\mu}^{P_{r}}\right) \cdot \rho_{r}^{\mu}\left(M, v^{P}\right)\right]$ $=\left[\rho_{i}^{\mu}\left(P_{r}, v_{\mu}^{P_{r}}\right) \cdot \rho_{r}^{\mu}\left(M, v^{P}\right)\right]=\psi_{i}^{\mu}(N, v, P)$.

By $i i i)$ of proposition 1 we know that $\psi_{i}^{\mu}(N, v, P)=\rho_{i}^{\mu}(N, v)$. Then $\Pi_{i}^{\mu}(N, v, P)=\psi_{i}^{\mu}(N, v, P)=\rho_{i}^{\mu}(N, v)$. 
The first property is a generalization of the classical consistency property that we will discuss in the following section. The properties 2 and 3 show that $\Pi_{i}^{\mu}(N, v, P)$ is a generalization of $\psi_{i}^{\mu}(N, v, P)$ when $i$ belongs to more than one configurational coalition. Indeed, when $i$ belongs to only one configurational coalition $\left(\left|P^{i}\right|=1\right.$ for all $i \in N), \Pi_{i}^{\mu}(N, v, P)=\psi_{i}^{\mu}(N, v, P)$. Moreover, $\Pi_{i}^{\mu}(N, v, P)$ generalize $\rho_{i}^{\mu}(N, v)$. Indeed when there is only one configurational coalition in the coalition configuration, or when there are $n$ singleton configurational coalitions in the coalition configuration, $\rho^{\mu}(N, v)=\Pi^{\mu}(N, v,\{N\})=\Pi^{\mu}\left(N, v,\{i\}_{i \in N}\right)$.

The following proposition shows that $\Pi^{\mu}$ is the Owen-Shapley-CCF share function, $\Pi^{S h}$, when $\mu=\mu^{S h}$; whereas $\Pi^{\mu}$ is the Banzhaf-CCF share function, $\Pi^{B}$, when $\mu=\mu^{B}$.

Proposition 6. The general CCF-share function for games with coalition configuration $\Pi^{\mu}$ is

i) Owen-Shapley-CCF share function $\Pi^{S h}$ when $\mu(N, v)=\mu^{S h}(N, v)=v(N)$,

ii) Banzhaf-CCF share function $\Pi^{B}$ when $\mu(N, v)=\mu^{B}(N, v)=\frac{1}{2^{|N|-1}} \sum_{S \subseteq N}(2|S|-|N|) v(S)$.

Proof. i) We provided in Section 3, that $\rho_{i}^{S h}\left(P_{r}, v^{P_{r}}\right) \cdot \rho_{r}^{S h}\left(M, v^{P}\right)=\rho_{i}^{\mu^{S h}}\left(P_{r}, v_{\mu^{S h}}^{P_{r}}\right) \cdot \rho_{r}^{\mu^{S h}}\left(M, v^{P}\right)$. Since $\Pi_{i}^{S h}(N, v, P)$ $=\sum_{P_{r} \in P^{i}}\left[\rho_{i}^{S h}\left(P_{r}, v^{P_{r}}\right) \cdot \rho_{r}^{S h}\left(M, v^{P}\right)\right]$ and $\Pi_{i}^{\mu^{S h}}(N, v, P)=\sum_{P_{r} \in P^{i}}\left[\rho_{i}^{\mu^{S h}}\left(P_{r}, v_{\mu^{S h}}^{P_{r}}\right) \cdot \rho_{r}^{\mu^{S h}}\left(M, v^{P}\right)\right]$, then $\Pi_{i}^{S h}(N, v, P)=$ $\sum_{P_{r} \in P^{i}}\left[\rho_{i}^{S h}\left(P_{r}, v^{P_{r}}\right) \cdot \rho\left(M, v^{P}\right)\right]=\sum_{P_{r} \in P^{i}}\left[\rho_{i}^{\mu^{S h}}\left(P_{r}, v_{\mu^{S h}}^{P_{r}}\right) \cdot \rho_{r}^{\mu^{S h}}\left(M, v^{P}\right)\right]=\Pi_{i}^{\mu^{S h}}(N, v, P)$.

ii) In the same way, we prove that $\Pi_{i}^{B}(N, v, P)=\Pi_{i}^{\mu^{B}}(N, v, P)$.

We can obtain also a $C C F$-share function for Deegan-Packel, as we obtained $\psi_{i}^{\mu^{D P}}$ from $\psi^{\mu}$. This function denoted $\Pi_{i}^{\mu^{D P}}(N, v, P)$ is given by

$$
\Pi_{i}^{\mu^{D P}}(N, v, P)=\sum_{P_{r} \in P^{i}}\left[\rho_{i}^{\mu^{D P}}\left(P_{r}, v_{\mu}^{P_{r}}\right) \cdot \rho_{r}^{\mu^{D P}}\left(M, v^{P}\right)\right], i \in N .
$$

Example 1. Consider the monotone game $(N, v)$ with player set $N=\{1,2,3,4,5\}$ given by $v(S)=\left\{\begin{array}{ll}3 & \text { if }|S| \geq 3 \\ 1 & \text { if }|S|=2 \\ 0 & \text { otherwise }\end{array}\right.$.

Assume that players are organized into an a priori coalition configuration, such that $P=\left\{P_{1}, P_{2}, P_{3}\right\}$ with $P_{1}=\{1,2,3\}, P_{2}=\{1,4\}$ and $P_{3}=\{3,5\}$. Then the external game $\left(M, v^{P}\right)$ with player set $M=\left\{P_{1}, P_{2}, P_{3}\right\}$ is

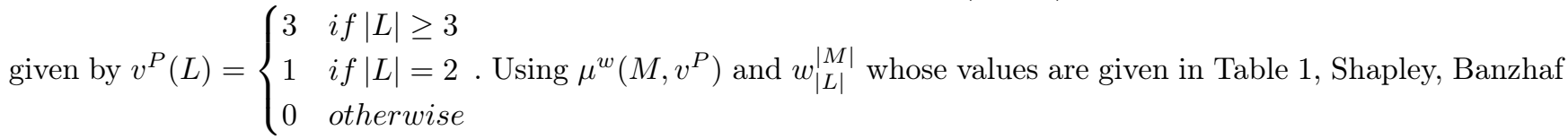
and Deegan-Packel shares of the players in this game are given by $\rho^{S h}\left(M, v^{P}\right)=\left(\frac{5}{9}, \frac{2}{9}, \frac{2}{9}\right), \rho^{B}\left(M, v^{P}\right)=\left(\frac{7}{13}, \frac{3}{13}, \frac{3}{13}\right)$ and $\rho^{\mu^{D P}}\left(M, v^{P}\right)=\left(\frac{29}{51}, \frac{11}{51}, \frac{11}{51}\right)$.

Now consider the shares of each player in the internal game corresponding to $P_{1}$. For $L \subseteq\left\{P_{2} P_{3}\right\} \subset M$, the marginal contribution $v^{P_{1}, L}(S), S \subseteq P_{1}$ is given in Table 3 . And given these values and the weighted vectors $\tilde{w}_{|L|}^{|3|}=\left(\frac{1}{3}, \frac{1}{6}, \frac{1}{3}\right), \bar{w}_{|L|}^{|3|}=\left(\frac{1}{4}, \frac{1}{4}, \frac{1}{4}\right)$ and $\hat{w}_{|L|}^{|3|}=\left(\frac{7}{3}, \frac{2}{3}, \frac{1}{3}\right)$, we obtain in Table 4 the characteristic function $v_{\mu}^{P_{1}}(S)$. And with $w_{|S|}^{\left|P_{1}\right|}$ and $\mu^{w}\left(P_{1}, v_{\mu}^{P_{1}}\right)$ as given in Table 2 , we obtain $\rho^{S h}\left(P_{1}, v_{\mu^{S h}}^{P_{1}}\right)=\left(\frac{3}{10}, \frac{2}{5}, \frac{3}{10}\right), \rho^{B}\left(P_{1}, v_{\mu^{B}}^{P_{1}}\right)=\left(\frac{2}{7}, \frac{3}{7}, \frac{2}{7}\right)$ and $\rho^{\mu^{D P}}\left(P_{1}, v_{\mu^{D P}}^{P_{1}}\right)=\left(\frac{13}{45}, \frac{19}{45}, \frac{13}{45}\right)$.

Similarly, for the internal game corresponding to $P_{2},\left(P_{2}, v_{\mu}^{P_{2}}\right)$, we have $\rho^{S h}\left(P_{2}, v_{\mu^{S h}}^{P_{2}}\right)=\rho^{B}\left(P_{2}, v_{\mu^{B}}^{P_{2}}\right)=$ $\rho^{\mu^{D P}}\left(P_{2}, v_{\mu^{D P}}^{P_{2}}\right)=\left(\frac{1}{2}, \frac{1}{2}\right)$. And for $\left(P_{3}, v_{\mu}^{P_{3}}\right)$, the internal game corresponding to $P_{3}, \rho^{S h}\left(P_{3}, v_{\mu^{S h}}^{P_{3}}\right)=\rho^{B}\left(P_{3}, v_{\mu^{B}}^{P_{3}}\right)=$ $\rho^{\mu^{D P}}\left(P_{3}, v_{\mu^{D P}}^{P_{3}}\right)=\left(\frac{1}{2}, \frac{1}{2}\right)$.

Finally, for the Owen-Shapley-CCF share function, the Banzhaf-CCF share function and Owen-Deegan-PackelCCF share function, we have respectively, $\Pi^{S h}(N, v, P)=\left(\frac{5}{18}, \frac{2}{9}, \frac{5}{18}, \frac{1}{9}, \frac{1}{9}\right), \Pi^{B}(N, v, P)=\left(\frac{7}{26}, \frac{3}{13}, \frac{7}{26}, \frac{3}{26}, \frac{3}{26}\right)$ and $\Pi^{\mu^{D P}}(N, v, P)=\left(\frac{1249}{4590}, \frac{551}{2295}, \frac{1249}{102}, \frac{11}{102}, \frac{11}{102}\right)$. Note that the share of player 3 , which belongs to more than one coalition, is equal to the sum of the share he receives in coalition $P_{1}$ and the share he receives in coalition $P_{3}$. For example $\Pi_{3}^{S h}(N, v, P)=\left[\rho_{3}^{S h}\left(P_{1}, v_{\mu^{S h}}^{P_{1}}\right) * \rho_{P_{1}}^{S h}\left(M, v^{P}\right)\right]+\left[\rho_{3}^{S h}\left(P_{3}, v_{\mu^{S h}}^{P_{3}}\right) * \rho_{P_{3}}^{S h}\left(M, v^{P}\right)\right]=\frac{3}{10} * \frac{5}{9}+\frac{1}{2} * \frac{2}{9}=\frac{5}{18}$. 


\begin{tabular}{|c|c|c|}
\hline Table 1 & \multicolumn{2}{|c|}{ Weight vectors } \\
\hline & $w_{|L|}^{|M|}$ & $\mu^{w}\left(M, v^{P}\right)$ \\
\hline Shapley & $\left(\frac{1}{3}, \frac{1}{6}, \frac{1}{3}\right)$ & 3 \\
\hline Banzhaf & $\left(\frac{1}{4}, \frac{1}{4}, \frac{1}{4}\right)$ & $\frac{13}{4}$ \\
\hline Deegan & $\left(\frac{7}{3}, \frac{2}{3}, \frac{1}{3}\right)$ & 17 \\
\hline
\end{tabular}

Table 2
\begin{tabular}{|c|c|c|}
\hline & $w_{|S|}^{\left|P_{1}\right|}$ & $\mu^{w}\left(P_{1}, v_{\mu}^{P_{1}}\right)$ \\
\hline Shapley & $\left(\frac{1}{3}, \frac{1}{6}, \frac{1}{3}\right)$ & $\frac{5}{3}$ \\
\hline Banzhaf & $\left(\frac{1}{4}, \frac{1}{4}, \frac{1}{4}\right)$ & $\frac{7}{4}$ \\
\hline Deegan & $\left(\frac{7}{3}, \frac{2}{3}, \frac{1}{3}\right)$ & 30 \\
\hline
\end{tabular}

Table 3 Marginal Contributions
\begin{tabular}{|c|c|c|c|c|}
\hline \multicolumn{5}{|c|}{$v^{P_{1}, L}(S), L \subseteq\left\{P_{2} P_{3}\right\} \subset M, S \subseteq P_{1}$} \\
\hline \hline$S \subseteq P_{1}$ & $v^{P_{1}, \varnothing}(S)$ & $v^{P_{1}, P_{2}}(S)$ & $v^{P_{1}, P_{3}}(S)$ & $v^{P_{1}, P_{2} P_{3}}(S)$ \\
\hline$\{\varnothing\}$ & 0 & 0 & 0 & 0 \\
\hline$\{1\}$ & 0 & 0 & 2 & 0 \\
\hline$\{2\}$ & 0 & 2 & 2 & 0 \\
\hline$\{3\}$ & 0 & 2 & 0 & 0 \\
\hline$\{1,2\}$ & 1 & 2 & 2 & 0 \\
\hline$\{1,3\}$ & 1 & 2 & 2 & 0 \\
\hline$\{2,3\}$ & 1 & 2 & 2 & 0 \\
\hline$\{1,2,3\}$ & 3 & 2 & 2 & 0 \\
\hline
\end{tabular}

Table 4 Characteristic functions of the internal games

\begin{tabular}{|c|c|c|c|}
\hline \multicolumn{3}{|c|}{$v_{\mu}^{P_{1}}(S)$} \\
\hline$S$ & Shapley & Banzhaf & Deegan \\
\hline$\{\varnothing\}$ & 0 & 0 & 0 \\
\hline$\{1\}$ & $\frac{1}{3}$ & $\frac{1}{2}$ & $\frac{4}{3}$ \\
\hline$\{2\}$ & $\frac{2}{3}$ & 1 & $\frac{8}{3}$ \\
\hline$\{3\}$ & $\frac{1}{3}$ & $\frac{1}{2}$ & $\frac{4}{3}$ \\
\hline$\{1,2\}$ & 1 & $\frac{5}{4}$ & 5 \\
\hline$\{1,3\}$ & 1 & $\frac{5}{4}$ & 5 \\
\hline$\{2,3\}$ & 1 & $\frac{5}{4}$ & 5 \\
\hline$\{1,2,3\}$ & $\frac{5}{3}$ & $\frac{7}{24}$ & $\frac{29}{3}$ \\
\hline
\end{tabular}

\section{An axiomatization of the class of share functions for games with coali- tion configuration}

In this section we present five axioms which characterize the general $C C F$-share function $\Pi^{\mu}$. In the following axioms, let $\mu$ be a symmetric, positive and additive function on $\mathcal{G}$ and $\Pi^{\mu}$ a $C C F$-share function on $\mathcal{G} \mathcal{P}^{\mathcal{N}}$.

The first axiom is the axiom of "generalized consistency". This axiom generalizes to games with coalition configuration the axiom introduced by van der Laan and van den Brink (2002) and van den Brink and van der Laan (2005) to characterize the class of share functions for games with coalition structure. A share function is consistent if the total payoff given by the share to the players in a configurational coalition $P_{k}$ is equal to the share of this coalition in the external game.

Generalized consistency : If $(N, v, P) \in \mathcal{G} \mathcal{P}^{\mathcal{N}}$ and $P_{k} \in P$, then:

$$
\sum_{i \in P_{k}} \Pi_{i}^{\mu}(N, v, P)=\sum_{i \in P_{k}}\left(\sum_{P_{r} \in P^{i}}\left(\rho_{i}^{\mu}\left(P_{r}, v_{\mu}^{P_{r}}\right) \cdot \rho_{r}^{\mu}\left(M, v^{P}\right)\right)\right) .
$$

The second axiom is the "null player property". The null player property only requires that a null player in a non-null game earns a zero payoff. In other word, the share of such a player is zero.

Null player property: If $i \in N$ is a null player in $(N, v) \in \mathcal{G}^{+}(i . e . v(S)=v(S \backslash\{i\})$ for all $S \subseteq N)$ then

$$
\Pi_{i}^{\mu}(N, v, P)=0 \text { for every } P \in \mathcal{P}^{N} \text {. }
$$

The third axiom is the "individual symmetry*". By comparison with the classical axiom of individual symmetry which is used to characterize values for games with coalition structure, with games with coalition configuration we must add a condition. The classical individual symmetry axiom states that players who belong to the same a priori coalition and are symmetric in the game earn the same share in the payoff. But as a player can belong to more than one a priori coalition in a game with coalition configuration, we add the condition that the set of configurational coalitions to which $i$ belongs is equal to the set of configurational coalitions to which $j$ belongs, and it is only under this condition that the share they receive is equal. If $i$ and $j$ belong to only one configurational coalition, then we have the classical axiom of symmetry.

Individual symmetry*: If $i, j \in N$ are symmetric in $(N, v) \in \mathcal{G}$ and $P^{i}=P^{j}$, then

$$
\Pi_{i}^{\mu}(N, v, P)=\Pi_{j}^{\mu}(N, v, P) .
$$

The next axiom is a generalization of the axiom of " $\mu$-additivity for coalition structures", introduced by van der Laan and van den Brink (2002) and van den Brink and van der Laan (2005) in order to characterize the general 
$C S$-share function. According to this axiom, if we sum two games with coalition configuration then the shares in the sum game is a specific convex combination of the shares in the separate games.

$\mu$-additivity for coalition configuration: Let $\mu: \mathcal{G} \rightarrow \mathbb{R}$ be given. For $(N, v, P),(N, w, P) \in \mathcal{G} \mathcal{P}^{N}$ let the function $z$ be given by $z=v+w$. For $i \in P_{r} \in P^{i}$, it holds that $\Pi_{i}^{\mu}(N, v+w, P)=\sum_{P_{r} \in P^{i}}$

$$
\left[\frac{\left[\mu\left(P_{r}, v_{\mu}^{P_{r}}\right) \Pi_{i}^{\mu}\left(P_{r}, v_{\mu}^{P_{r}},\left\{P_{r}\right\}\right)+\mu\left(P_{r}, w_{\mu}^{P_{r}}\right) \Pi_{i}^{\mu}\left(P_{r}, w_{\mu}^{P_{r}},\left\{P_{r}\right\}\right)\right] *\left[\mu\left(M, v^{P}\right) \Pi_{P_{r}}^{\mu}\left(M, v^{P},\{M\}\right)+\mu\left(M, w^{P}\right) \Pi_{P_{r}}^{\mu}\left(M, w^{P},\{M\}\right)\right]}{\left[\mu\left(P_{r}, v_{\mu}^{P_{r}}\right)+\mu\left(P_{r}, w_{\mu}^{P_{r}}\right)\right] *\left[\mu\left(M, v^{P}\right)+\mu\left(M, w^{P}\right)\right]}\right] .
$$

These first four axioms are axioms used by van der Laan and van den Brink (2002) and van den Brink and van der Laan (2005) to characterize the class of share functions for games with coalition structure. Indeed, they proved that $\psi^{\mu}$ is the unique share function that satisfies these four axioms. The last axiom is an axiom introduced by Albizuri et al. (2006a, 2006b) to characterize values for games with coalition configuration.

To introduce the last axiom we need the following notations and definitions.

Let $i, j \in N$. Given $v \in \mathcal{G}$, we denote $v_{i \backslash j}$ the game on $N \backslash j$ defined by

$$
v_{i \backslash j}(S)=\left\{\begin{array}{c}
v(S) \text { if } i \notin S \\
v(S \cup j) \text { if } i \in S
\end{array} \mid .\right.
$$

Let $P \in \mathcal{P}^{N}$, such that $P^{i} \cap P^{j}=\varnothing$ (i.e. $i$ does not belong to configurational coalitions to which $j$ belongs), we denote by $P_{i \backslash j}$ the coalition configuration on $N \backslash j$ defined by,

$$
P_{i \backslash j}=\left(P \backslash P^{j}\right) \cup\left\{\left(P_{r} \backslash\{j\}\right) \cup\{i\}: P_{r} \in P^{j}\right\} .
$$

That is in $P_{i \backslash j}$ and $v_{i \backslash j}$, the players $i$ and $j$ have been amalgamated into a single player, player $i$. We can say that player $j$ has abandoned the game and now player $i$ represents both players in the new situations.

We say that $i, j \in N$ are doubles in $(N, v, P) \in \mathcal{G P} \mathcal{P}^{N}$ if:

i) $v(S \cup j)=v(S \cup i)$ for every $S \subset N \backslash\{i, j\}$ and;

ii) $S \cup\{i\} \in P^{i} \rightarrow S \cup\{j\}, S \cup\{i, j\} \notin P^{j}$, for every $S \subset N \backslash\{i, j\}$.

Notice that if $i$ and $j$ are doubles then they can be understood as if they were representing a unique player. In other words, when one of these players joins a coalition he produces the same effect as if both of them do it. This is reflected in the second condition: if a coalition joins player $i$, then this coalition does not need to join player $j$. That is, players $\mathrm{i}$ and $\mathrm{j}$ cannot share exactly the same partners nor be together in the same member of the coalition configuration.

We can now introduce the last axiom.

Merger: If $i, j \in N$ are doubles in $(N, v, P) \in \mathcal{G P}^{N}$, and $P^{i} \cap P^{j}=\varnothing$, then for every $z \in N \backslash\{i, j\}$, it holds that:

$$
\Pi_{z}^{\mu}(N, v, P)=\Pi_{z}^{\mu}\left(N, v_{i \backslash j}, P_{i \backslash j}\right)
$$

Imagine that $j$ leaves the game, and that $i$ represents him: $i$ will play in the game as if acting as proxy for $j$ in some coalitions, then $i$ and $j$ are doubles. According to the merger axiom, that should not have any influence on the payments to the other players. In other words, if $i$ and $j$ are doubles, when they merge this axiom requires that the share does not change for players other than $i$ and $j$.

Note that the merger axiom introduced by Albizuri et al. (2006a, 2006b) is very close to well-known merging properties in TU-games, such as those in Lehrer (1988) and Haller (1994). ${ }^{10}$ Lehrer (1988) used "amalgamation axioms" to characterize the Banzhaf value, whereas Haller (1994) characterized the Banzhaf value with "collusion axioms". The merger axiom can be seen as an adaptation to the coalitional configuration framework of these two axioms.

Theorem 1. Let $\mu: \mathcal{G} \rightarrow \mathbb{R}$ be additive, positive and symmetric on $\mathcal{G}$. Then $\Pi^{\mu}$ is the unique $\mathrm{CCF}$-share function on $\mathcal{G}{ }^{N}$ that satisfies generalized consistency, null player, individual symmetry, $\mu$-additivity for coalition configuration and merger.

Let us prove first that $\Pi^{\mu}$ verify all these axioms.

Proof. I) Consistency: This property is already proved in Proposition 6.

II) Null player: Let $i \in P_{r} \in P^{i}$ be a null player in $(N, v) \in \mathcal{G}^{+}$. Then $v_{P_{r} \mid S}^{P}(L)=v_{P_{r} \mid S \backslash\{i\}}^{P}(L)$ for all $L \subseteq M$, therefore $\mu\left(M, v_{P_{r} \mid S}^{P}\right)=\mu\left(M, v_{P_{r} \mid S \backslash\{i\}}^{P}\right)$ for all $S \subseteq P_{r}, i \in S$. This implies that $v_{\mu}^{P_{r}}(S)-v_{\mu}^{P_{r}}(S \backslash\{i\})$

\footnotetext{
${ }^{10}$ Section 6 in Haller (1994) deals with the relationship and the difference between amalgamation and collusion properties.
} 
$=\rho_{r}^{\mu}\left(M, v_{P_{r} \mid S}^{P}\right) \mu\left(M, v_{P_{r} \mid S}^{P}\right)-\rho_{r}^{\mu}\left(M, v_{P_{r} \mid S \backslash\{i\}}^{P}\right) \mu\left(M, v_{P_{r} \mid S \backslash\{i\}}^{P}\right)=0$, for all $S \subseteq P_{r}, i \in S$. Then $v_{\mu}^{P_{r}}(S)=v_{\mu}^{P_{r}}(S \backslash\{i\})$ and $i$ is a null player in $\left(P_{r}, v_{\mu}^{P_{r}}\right)$. As $\rho$ verifies the property of null player (see van der Laan and van den Brink 1998), then $\rho_{i}\left(P_{r}, v_{\mu}^{P_{r}}\right)=0$, and $\Pi_{i}^{\mu}(N, v, P)=\sum_{P_{r} \in P^{i}}\left[\rho_{i}^{\mu}\left(P_{r}, v_{\mu}^{P_{r}}\right) \cdot \rho_{r}^{\mu}\left(M, v^{P}\right)\right]=0$ if $v_{\mu}^{P_{r}} \in \mathcal{G}^{+}$.

Now if $\left(P_{r}, v_{\mu}^{P_{r}}\right)$ is a null game, then $v_{\mu}^{P_{r}}(S)=\rho_{r}^{\mu}\left(M, v_{P_{r} \mid S}^{P}\right) \mu\left(M, v_{P_{r} \mid S}^{P}\right)=0$. Consequently $v_{\mu}^{P_{r}}\left(P_{r}\right)$ $=\rho_{r}^{\mu}\left(M, v_{P_{r} \mid P_{r}}^{P}\right) \mu\left(M, v_{P_{r} \mid P_{r}}^{P}\right)=0$. And since $\mu\left(M, v_{P_{r} \mid P_{r}}^{P}\right)$ is positive by definition, then $\rho_{r}^{\mu}\left(M, v_{P_{r} \mid P_{r}}^{P}\right)=0$. Since $v_{P_{r} \mid P_{r}}^{P}=v(P(L))=v^{P}(L)$, then $\rho_{r}^{\mu}\left(M, v_{P_{r} \mid P_{r}}^{P}\right)=\rho_{r}^{\mu}\left(M, v^{P}\right)=0$. Again $\Pi_{i}^{\mu}(N, v, P)=\sum_{P_{r} \in P^{i}}\left[\rho_{i}^{\mu}\left(P_{r}, v_{\mu}^{P_{r}}\right) \cdot \rho_{r}^{\mu}\left(M, v^{P}\right)\right]$ $=0$, and $\Pi^{\mu}$ satisfies the null player property.

III) Individual Symmetry: If $i$ and $j$ are symmetric players in $(N, v)$, then $i$ and $j \in P_{r}$ are symmetric in $\left(P_{r}, v_{\mu}^{P_{r}}\right)$. Since $\rho^{\mu}$ is symmetric (van der Laan and van den Brink 1998), then $\rho_{i}^{\mu}\left(P_{r}, v_{\mu}^{P_{r}}\right) . \rho_{r}^{\mu}\left(M, v^{P}\right)$ $=\rho_{j}^{\mu}\left(P_{r}, v_{\mu}^{P_{r}}\right) \cdot \rho_{r}^{\mu}\left(M, v^{P}\right)$. We must then distinguish two cases:

a) If $i$ and $j$ belong to one configurational coalition, namely $P_{r},\left|P^{i}\right|=\left|P^{j}\right|=1$, then $\Pi_{i}^{\mu}(N, v, P)$ $=\sum_{P_{r} \in P^{i}}\left[\rho_{i}^{\mu}\left(P_{r}, v_{\mu}^{P_{r}}\right) \cdot \rho_{r}^{\mu}\left(M, v^{P}\right)\right]=\rho_{i}^{\mu}\left(P_{r}, v_{\mu}^{P_{r}}\right) \cdot \rho_{r}^{\mu}\left(M, v^{P}\right)$ and $\Pi_{j}^{\mu}(N, v, P)=\sum_{P_{r} \in P^{j}}\left[\rho_{j}^{\mu}\left(P_{r}, v_{\mu}^{P_{r}}\right) \cdot \rho_{r}^{\mu}\left(M, v^{P}\right)\right]$ $=\rho_{j}^{\mu}\left(P_{r}, v_{\mu}^{P_{r}}\right) \cdot \rho_{r}^{\mu}\left(M, v^{P}\right)$. Since $\rho_{i}^{\mu}\left(P_{r}, v_{\mu}^{P_{r}}\right) \cdot \rho_{r}^{\mu}\left(M, v^{P}\right)=\rho_{j}^{\mu}\left(P_{r}, v_{\mu}^{P_{r}}\right) \cdot \rho_{r}^{\mu}\left(M, v^{P}\right)$, then $\Pi_{i}^{\mu}(N, v, P)=\Pi_{j}^{\mu}(N, v, P)$.

b) If $i$ and $j$ belongs to more than one configurational coalition, by definition of the property $P^{i}=P^{j}$, then $\sum_{P_{r} \in P^{i}}\left[\rho_{i}^{\mu}\left(P_{r}, v_{\mu}^{P_{r}}\right) . \rho_{r}^{\mu}\left(M, v^{P}\right)\right]=\sum_{P_{r} \in P^{j}}\left[\rho_{j}^{\mu}\left(P_{r}, v_{\mu}^{P_{r}}\right) \cdot \rho_{r}^{\mu}\left(M, v^{P}\right)\right] \Leftrightarrow \Pi_{i}^{\mu}(N, v, P)=\Pi_{j}^{\mu}(N, v, P)$. And $\Pi^{\mu}$ satisfies individual symmetry*.

IV) $\mu$-additivity: We must distinguish three cases:

1) We suppose first that $(N, z)$ is a null game. Since $z=v^{0},\left(P_{r}, z_{\mu}^{P_{r}}\right)$ and $\left(M, z^{P}\right)$ are null games. And since $z=v^{0}$ if and only if $v=w=v^{0}$, then $\left(P_{r}, z_{\mu}^{P_{r}}\right)$ as a null game implies that $\left(P_{r}, v_{\mu}^{P_{r}}\right)$ and $\left(P_{r}, w_{\mu}^{P_{r}}\right)$ are null games. Similarly $\left(M, z^{P}\right)$ null game implies that $\left(M, v^{P}\right)$ and $\left(M, w^{P}\right)$ are null games. Therefore the $\mu$-additivity is true.

2) Assume now that $(N, z) \in \mathcal{G}^{+}$and $i \in P_{r} \in P$ is a null player in $(N, z)$. Then he is also a null player in $(N, v)$ and $(N, w)$ because these games are both monotonic games. With the same arguments as above for the null player axiom, we show that $\Pi^{\mu}(N, z, P)=0$ and that $i$ is a null player in $\left(P_{r}, v_{\mu}^{P_{r}}\right)$. If $v_{\mu}^{P_{r}}=v^{0}$, then $\mu\left(P_{r}, v_{\mu}^{P_{r}}\right)=0$, and if $v_{\mu}^{P_{r}} \neq v^{0}$, since $i$ is a null player in $\left(P_{r}, v_{\mu}^{P_{r}}\right), \rho_{i}^{\mu}\left(P_{r}, v_{\mu}^{P_{r}}\right)=0$. In these two cases $\mu\left(P_{r}, v_{\mu}^{P_{r}}\right) \rho_{i}^{\mu}\left(P_{r}, v_{\mu}^{P_{r}}\right)=0$. In the same way we show that $\mu\left(P_{r}, w_{\mu}^{P_{r}}\right) \rho_{i}^{\mu}\left(P_{r}, w_{\mu}^{P_{r}}\right)=0$. Therefore $\mu$-additivity is verified.

3) To conclude, consider the case that $i$ is not a null player. In that case $\mu\left(P_{r}, z_{\mu}^{P_{r}}\right) \mu\left(M, z^{P}\right)>0$, then $\mu\left(P_{r}, z_{\mu}^{P_{r}}\right) \mu\left(M, z^{P}\right) \Pi_{i}^{\mu}(N, z, P)=\mu\left(P_{r}, z_{\mu}^{P_{r}}\right) \mu\left(M, z^{P}\right) \sum_{P_{r} \in P^{i}}\left[\rho_{i}^{\mu}\left(P_{r}, z_{\mu}^{P_{r}}\right) \cdot \rho_{r}^{\mu}\left(M, z^{P}\right)\right]$. And by the $\mu$-additivity of $\rho^{\mu}$ (van der Laan and van den Brink 1998), we have: $\rho_{i}^{\mu}\left(P_{r}, z_{\mu}^{P_{r}}\right)=\frac{\left[\mu\left(P_{r}, v_{\mu}^{P_{r}}\right) \rho_{i}^{\mu}\left(P_{r}, v_{\mu}^{P_{r}}\right)\right]+\left[\mu\left(P_{r}, w_{\mu}^{P_{r}}\right) \rho_{i}^{\mu}\left(P_{r}, w_{\mu}^{P_{r}}\right)\right]}{\left[\mu\left(P_{r}, z_{\mu}^{P_{r}}\right)\right]}$ and $\rho_{r}^{\mu}\left(M, z^{P}\right)=\frac{\left[\mu\left(M, v^{P}\right) \rho_{r}^{\mu}\left(M, v^{P}\right)\right]+\left[\mu\left(M, w^{P}\right) \rho_{r}^{\mu}\left(M, w^{P}\right)\right]}{\left[\mu\left(M, z^{P}\right)\right]}$.

Then $\mu\left(P_{r}, z_{\mu}^{P_{r}}\right) \mu\left(M, z^{P}\right)\left[\sum_{P_{r} \in P^{i}} \rho_{i}^{\mu}\left(P_{r}, z_{\mu}^{P_{r}}\right) \cdot \rho_{r}^{\mu}\left(M, z^{P}\right)\right]=\mu\left(P_{r}, z_{\mu}^{P_{r}}\right) \mu\left(M, z^{P}\right)$

$\sum_{P_{r} \in P^{i}}\left(\frac{\left[\mu\left(P_{r}, v_{\mu}^{P_{r}}\right) \rho_{i}^{\mu}\left(P_{r}, v_{\mu}^{P_{r}}\right)\right]+\left[\mu\left(P_{r}, w_{\mu}^{P_{r}}\right) \rho_{i}^{\mu}\left(P_{r}, w_{\mu}^{P_{r}}\right)\right]}{\left[\mu\left(P_{r}, z_{\mu}^{P_{r}}\right)\right]} * \frac{\left[\mu\left(M, v^{P}\right) \rho_{r}^{\mu}\left(M, v^{P}\right)\right]+\left[\mu\left(M, w^{P}\right) \rho_{r}^{\mu}\left(M, w^{P}\right)\right]}{\left[\mu\left(M, z^{P}\right)\right]}\right)=$ $\sum_{P_{r} \in P^{i}}\left(\left[\mu\left(P_{r}, v_{\mu}^{P_{r}}\right) \rho_{i}^{\mu}\left(P_{r}, v_{\mu}^{P_{r}}\right)+\mu\left(P_{r}, w_{\mu}^{P_{r}}\right) \rho_{i}^{\mu}\left(P_{r}, w_{\mu}^{P_{r}}\right)\right] *\left[\mu\left(M, v^{P}\right) \rho_{r}^{\mu}\left(M, v^{P}\right)+\mu\left(M, w^{P}\right) \rho_{r}^{\mu}\left(M, w^{P}\right)\right]\right)$. And from the consistency property, $\mu\left(P_{r}, z_{\mu}^{P_{r}}\right) \mu\left(M, z^{P}\right) \Pi_{i}^{\mu}(N, z, P)=$ $\sum_{P_{r} \in P^{i}}\left(\begin{array}{c}{\left[\mu\left(P_{r}, v_{\mu}^{P_{r}}\right) \Pi_{i}^{\mu}\left(P_{r}, v_{\mu}^{P_{r}},\left\{P_{r}\right\}\right)+\mu\left(P_{r}, w_{\mu}^{P_{r}}\right) \Pi_{i}^{\mu}\left(P_{r}, w_{\mu}^{P_{r}},\left\{P_{r}\right\}\right)\right]} \\ *\left[\mu\left(M, v^{P}\right) \Pi_{P_{r}}^{\mu}\left(M, v^{P},\{M\}\right)+\mu\left(M, w^{P}\right) \Pi_{P_{r}}^{\mu}\left(M, w^{P},\{M\}\right)\right]\end{array}\right)$.

Moreover since $\mu: \mathcal{G} \rightarrow \mathbb{R}$ is additive and positive, then

$\Pi^{\mu}(N, v+w, P)=\sum_{P_{r} \in P^{i}}$ $\left[\frac{\left[\mu\left(P_{r}, v_{\mu}^{P_{r}}\right) \Pi_{i}^{\mu}\left(P_{r}, v_{\mu}^{P_{r}},\left\{P_{r}\right\}\right)+\mu\left(P_{r}, w_{\mu}^{P_{r}}\right) \Pi_{i}^{\mu}\left(P_{r}, w_{\mu}^{P_{r}},\left\{P_{r}\right\}\right)\right] *\left[\mu\left(M, v^{P}\right) \Pi_{P_{r}}^{\mu}\left(M, v^{P},\{M\}\right)+\mu\left(M, w^{P}\right) \Pi_{P_{r}}^{\mu}\left(M, w^{P},\{M\}\right)\right]}{\left[\mu\left(P_{r}, v_{\mu}^{P_{r}}\right)+\mu\left(P_{r}, w_{\mu}^{P_{r}}\right)\right] *\left[\mu\left(M, v^{P}\right)+\mu\left(M, w^{P}\right)\right]}\right]$. The $\mu$-additivity for coalition configuration is satisfied.

V) Merger: Let $v \in \mathcal{G}$ and $P \in \mathcal{P}^{N}$ and let $i$ and $j \in N$ be doubles in $(N, v, P) \in \mathcal{G P}^{N}$. We are going to prove that for all $z \in N \backslash\{i, j\}, \Pi_{z}^{\mu}(N, v, P)=\sum_{P_{r} \in P^{z}}\left[\rho_{z}^{\mu}\left(P_{r}, v_{\mu}^{P_{r}}\right) . \quad \rho_{r}^{\mu}\left(M, v^{P}\right)\right]=\Pi_{z}^{\mu}\left(N, v_{i \backslash j}, P_{i \backslash j}\right)$ $=\sum_{P_{r} \in P^{z}}\left[\rho_{z}^{\mu}\left(P_{r}, v_{i \backslash j_{\mu}}^{P_{r}}\right) \cdot \rho_{r}^{\mu}\left(M_{i \backslash j}, v_{i \backslash j}^{P}\right)\right]$. In order to do that, we proceed in three steps:

i) We first prove that $v^{P}(L)=v_{i \backslash j}^{P}(L)$ and therefore that $\rho_{r}^{\mu}\left(M_{i \backslash j}, v_{i \backslash j}^{P}\right)=\rho_{r}^{\mu}\left(M, v^{P}\right)$. 
Let $P=\left\{P_{1}, \ldots, P_{m}\right\}$ and $P_{i \backslash j}=\left(P \backslash P^{j}\right) \cup\left\{\left(P_{r} \backslash\{j\}\right) \cup\{i\}: P_{r} \in P^{j}\right\}$. That is in $P_{i \backslash j}$ players $i$ and $j$ are amalgamated into a same player. But this does not influence the number of configurational coalitions, there-

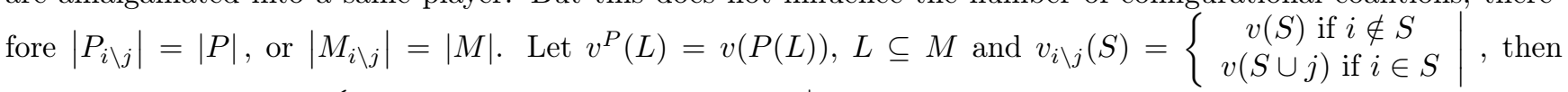
$v_{i \backslash j}^{P}(L)=v_{i \backslash j}(P(L))=\left\{\begin{array}{c}v(P(L)) \text { if } i \notin P(L) \subset M_{i \backslash j} \\ v(P(L) \cup j) \text { if } i \in P(L) \subseteq M_{i \backslash j}\end{array} \mid\right.$. Since $i$ and $j$ are doubles, $v(P(L) \cup j)=v(P(L) \cup i)$. Then $v_{i \backslash j}^{P}(L)=\left\{\begin{array}{c}v(P(L)) \text { if } i \notin P(L) \subset M_{i \backslash j} \\ v(P(L) \cup j) \text { if } i \in P(L) \subseteq M_{i \backslash j}\end{array} \mid=\left\{\begin{array}{c}v(P(L)) \text { if } i \notin P(L) \subset M_{i \backslash j} \\ v(P(L) \cup i) \text { if } i \in P(L) \subseteq M_{i \backslash j}\end{array} \mid\right.\right.$. Since $i \in P(L)$ when $v_{i \backslash j}^{P}(L)=v(P(L) \cup i)$, then $v(P(L) \cup i)=v(P(L))$ and $v_{i \backslash j}^{P}(L)=v^{P}(L)$. And since $i$ and $j$ are doubles $(v(S \cup j)=v(S \cup i))$, when $j$ is replaced by $i$ payments in the external game are identical, then $\rho_{r}^{\mu}\left(M, v^{P}\right)=$ $\rho_{r}^{\mu}\left(M, v_{i \backslash j}^{P}\right)=\rho_{r}^{\mu}\left(M_{i \backslash j}, v_{i \backslash j}^{P}\right)$.

ii) We provide now that $v_{\mu}^{P_{r}}=v_{i \backslash j_{\mu}}^{P_{r}}$, and therefore that $\rho_{z}^{\mu}\left(P_{r}, v_{\mu}^{P_{r}}\right)=\rho_{z}^{\mu}\left(P_{r}, v_{i \backslash j_{\mu}}^{P_{r}}\right)$.

Let $i$ and $j$ doubles and $v_{\mu}^{P_{r}}(S)=\rho_{r}^{\mu}\left(M, v_{P_{r} \mid S}^{P}\right) \mu\left(M, v_{P_{r} \mid S}^{P}\right), S \subseteq P_{r}$ with $v_{P_{r} \mid S}^{P}(L)=\left\{\begin{array}{c}v(P(L \backslash\{r\}) \cup S) \text { if } r \in L \subset M \\ v(P(L)) \text { if } r \notin L \subseteq M\end{array} \mid\right.$. We have also $v_{i \backslash j_{\mu}}^{P_{r}}(S)=\left\{\begin{array}{c}v_{\mu}^{P_{r}}(S) \text { if } i \notin S(\alpha) \\ v_{\mu}^{P_{r}}(S \cup j) \text { if } i \in S(\beta)\end{array} \mid\right.$ by definition of $v_{i \backslash j}(S)$. Then for $(\alpha)$, we have $v_{i \backslash j_{\mu}}^{P_{r}}(S)=v_{\mu}^{P_{r}}(S)$ if $i \notin S$, then $\rho_{z}^{\mu}\left(P_{r}, v_{\mu}^{P_{r}}\right)=\rho_{z}^{\mu}\left(P_{r}, v_{i \backslash j_{\mu}}^{P_{r}}\right)$. And for $(\beta), v_{i \backslash j_{\mu}}^{P_{r}}(S)=v_{\mu}^{P_{r}}(S \cup j)$ if $i \in S$, and by the definition of $v_{\mu}^{P_{r}}$, $v_{\mu}^{P_{r}}(S \cup j)=\rho_{r}^{\mu}\left(M, v_{P_{r} \mid S \cup j}^{P}\right) \mu\left(M, v_{P_{r} \mid S \cup j}^{P}\right), S \subseteq P_{r}$. With $v_{P_{r} \mid S \cup j}^{P}(L)=\left\{\begin{array}{c}v(P(L \backslash\{r\}) \cup\{S \cup j\}) \text { if } r \in L \subset M \\ v(P(L)) \text { if } r \notin L \subseteq M\end{array}\right.$ $=\left\{\begin{array}{c}v(P(L \backslash\{r\} \cup S) \cup\{j\}) \text { if } r \in L \subseteq M \\ v(P(L)) \text { if } r \notin L \subseteq M\end{array} \mid\right.$.

Since $i$ and $j$ doubles, $v(P(L \backslash\{r\} \cup S) \cup\{j\})=v(P(L \backslash\{r\} \cup S) \cup\{i\})$. But here $i \in S$ (case $\beta$ ) then $v(P(L \backslash\{r\} \cup S) \cup\{i\})=v(P(L \backslash\{r\} \cup S))$. Therefore $v_{P_{r} \mid S \cup j}^{P}(L)=\left\{\begin{array}{c}v(P(L \backslash\{r\} \cup S)) \text { if } r \in L \subset M \\ v(P(L)) \text { if } r \notin L \subseteq M\end{array} \mid=v_{P_{r} \mid S}^{P}(L)\right.$. Consequently, $\mu\left(M, v_{P_{r} \mid S \cup j}^{P}\right)=\mu\left(M, v_{P_{r} \mid S}^{P}\right)$ and $\rho_{r}^{\mu}\left(M, v_{P_{r} \mid S \cup j}^{P}\right)=\rho_{r}^{\mu}\left(M, v_{P_{r} \mid S}^{P}\right)$, then $v_{\mu}^{P_{r}}(S \cup j)=v_{\mu}^{P_{r}}(S)$. Therefore $v_{\mu}^{P_{r}}=v_{i \backslash j_{\mu}}^{P_{r}}$ and $\rho_{z}^{\mu}\left(P_{r}, v_{\mu}^{P_{r}}\right)=\rho_{z}^{\mu}\left(P_{r}, v_{i \backslash j_{\mu}}^{P_{r}}\right)$.

iii) Finally we show that $\Pi_{z}^{\mu}(N, v, P)=\Pi_{z}^{\mu}\left(N, v_{i \backslash j}, P_{i \backslash j}\right)$.

Since $\rho_{r}^{\mu}\left(M, v^{P}\right)=\rho_{r}^{\mu}\left(M_{i \backslash j}, v_{i \backslash j}^{P}\right), \rho_{z}^{\mu}\left(P_{r}, v_{\mu}^{P_{r}}\right)=\rho_{z}^{\mu}\left(P_{r}, v_{i \backslash j_{\mu}}^{P_{r}}\right)$ and $P^{z}=P_{i \backslash j}^{z}$, then for all $z \in N \backslash\{i, j\}$, $\sum_{P_{r} \in P^{z}}\left[\rho_{z}^{\mu}\left(P_{r}, v_{\mu}^{P_{r}}\right) \cdot \rho_{r}^{\mu}\left(M, v^{P}\right)\right]=\sum_{P_{r} \in P^{z}}\left[\rho_{z}^{\mu}\left(P_{r}, v_{i \backslash j_{\mu}}^{P_{r}}\right) \cdot \rho_{r}^{\mu}\left(M_{i \backslash j}, v_{i \backslash j}^{P}\right)\right]$, and $\Pi_{z}^{\mu}(N, v, P)==\Pi_{z}^{\mu}\left(N, v_{i \backslash j}, P_{i \backslash j}\right)$.

Hence the axiom of merger is verified.

Let us now prove that $\Pi^{\mu}$ is uniquely determined by all of these axioms.

To show uniqueness, suppose that $\Pi^{\mu}$ satisfies the 5 axioms. We will prove that $\Pi_{a}^{\mu}$ is fully determined for every $a \in N$ and $(N, v, P) \in \mathcal{G} \mathcal{P}^{N}$ by induction on $\sum_{\substack{P_{q}, P_{l} \in P \\ P_{q} \neq P_{l}}}\left|P_{q} \cap P_{l}\right|$. In order to do that we present our argument in successive steps.

First step of the induction.

Let $(N, v, P) \in \mathcal{G} \mathcal{P}^{N}$ and $\sum_{\substack{P_{q}, P_{l} \in P \\ P_{q} \neq P_{l}}}\left|P_{q} \cap P_{l}\right|=0\left(P_{q} \cap P_{l}=\varnothing\right.$ for all $\left.P_{q}, P_{l} \in P, P_{q} \neq P_{l}\right)$. Then $P$ is a partition, and since $\Pi^{\mu}$ satisfies consistency, null player property, individual symmetry* ${ }^{*}$, and $\mu$-additivity for coalition configuration, then $\Pi^{\mu}$ coincide with the coalitional share $\psi^{\mu}$ (since $\psi^{\mu}$ is the unique share function that satisfies these four axioms), that is $\Pi_{a}^{\mu}(N, v, P)=\psi_{a}^{\mu}(N, v, P)$ and therefore $\Pi_{a}^{\mu}(N, v, P)$ is fully determined.

Second step of the induction.

Let $r \geq 0$, i.e. $r \in \mathbb{N} \cup 0$ and assume that $\Pi_{a}^{\mu}(N, v, P)$ is determined for every $(N, v, P) \in \mathcal{G P}^{N}$ such that $\sum_{\substack{P_{q}, P_{l} \in P \\ P_{q} \neq P_{l}}}\left|P_{q} \cap P_{l}\right| \leq r$

Third step of the induction.

We will prove that $\Pi_{a}^{\mu}$ is determined if $a \in N$ when $\sum_{\substack{P_{q}, P_{l} \in P \\ P_{q} \neq P_{l}}}\left|P_{q} \cap P_{l}\right|=r+1$. Let us set $a \in N$. We distinguish two cases:

a) There exists $j \neq a$, such that $j \in P_{q} \cap P_{l}$ for some $P_{q}, P_{l}$ and $P_{q} \neq P_{l}$. Then take $i \notin N$ and $w \in \mathcal{G}^{N \cup\{i\}}$ be 
defined for every $S \subseteq N \cup\{i\}$ by $w(S)=\left\{\begin{array}{c}v(S) \text { if } i \notin S \\ v((S \backslash\{i\}) \cup\{j\}) \text { if } i \in S\end{array} \mid\right.$, and consider also the coalition configuration on $N \cup i$ defined by $\hat{P}=\left(P \backslash\left\{P_{q}\right\}\right) \cup\left\{\left(P_{q} \backslash\{j\}\right) \cup\{i\}\right\}$, we can show:

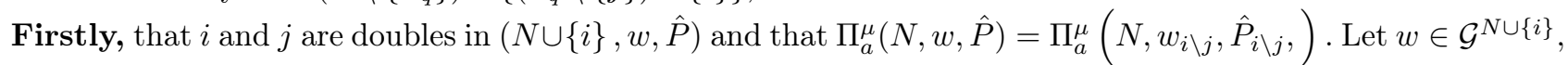
then $w(S \cup\{i\})=\left\{\begin{array}{c}v(S \cup\{i\}) \text { if } i \notin S \cup\{i\} \\ v(((S \cup\{i\}) \backslash\{i\}) \cup\{j\}) \text { if } i \in S \cup\{i\}\end{array} \mid=v((S \cup\{i\}) \backslash\{i\}) \cup\{j\}\right)$ since $i \in S \cup\{i\}$, then $w(S \cup\{i\})=v(S \cup\{j\})$. And $w(S \cup j\{\})=\left\{\begin{array}{c}v(S \cup\{j\}) \text { if } i \notin S \cup\{j\} \\ v(((S \cup\{j\}) \backslash\{i\}) \cup\{j\}) \text { if } i \in S \cup\{j\}\end{array} \mid=v(S \cup\{j\})\right.$ since $i \notin S \cup\{j\}$. Then $w(S \cup\{i\})=w(S \cup\{j\})$, and $i$ and $j$ are doubles in $w$. Merger says that for all $i$ and $j$ doubles, and for all $a \in N \backslash\{i, j\}$, we have $\Pi_{a}^{\mu}(N, v, P)=\Pi_{a}^{\mu}\left(N, v_{i \backslash j}, P_{i \backslash j},\right)$. Then $\Pi_{a}^{\mu}(N, w, \hat{P})=\Pi_{a}^{\mu}\left(N, w_{i \backslash j}, \hat{P}_{i \backslash j},\right)$.

Secondly, we can prove that $\Pi_{a}^{\mu}(N, w, \hat{P})=\Pi_{a}^{\mu}\left(N, w_{i \backslash j}, \hat{P}_{i \backslash j},\right)=\Pi_{a}^{\mu}(N, v, P)$. Let $v_{i \backslash j}(S)=\left\{\begin{array}{c}v(S) \text { if } i \notin S \\ v(S \cup\{j\}) \text { if } i \in S\end{array}\right.$ on $N \backslash\{j\}$ and $w_{i \backslash j}(S)=\left\{\begin{array}{c}w(S) \text { if } i \notin S \\ w(S \cup\{j\}) \text { if } i \in S\end{array} \mid\right.$. Since $i$ and $j$ are doubles in $w, w_{i \backslash j}(S)=\left\{\begin{array}{c}w(S) \text { if } i \notin S \\ w(S \cup\{i\}) \text { if } i \in S\end{array} \mid\right.$. By definition of $w, w(S)=v(S)$ if $i \notin S$ and $w(S \cup\{i\})=v(((S \cup\{i\}) \backslash\{i\}) \cup\{j\})=v(S \cup\{j\})$ if $i \in S$. Then $w_{i \backslash j}(S)=\left\{\begin{array}{c}v(S) \text { if } i \notin S \\ v(S \cup\{j\}) \text { if } i \in S\end{array} \mid=v_{i \backslash j}(S)\right.$. Let $P_{i \backslash j}=\left(P \backslash P^{j}\right) \cup\left\{\left(P_{r} \backslash\{j\}\right) \cup\{i\}: P_{r} \in P^{j}\right\}$, where in all the configurational coalitions containing $j, j$ is replaced by $i$. And let $\hat{P}=\left(P \backslash\left\{P_{q}\right\}\right) \cup\left\{P_{q} \backslash\{j\} \cup\{i\}\right\}$, where in $P_{q}, j$ is replaced by $i$. Then $\hat{P}$ is a particular case of $P_{i \backslash j}$, then $\hat{P} \subseteq P_{i \backslash j}$ and $\hat{P}_{i \backslash j}=P_{i \backslash j}$. Therefore $\Pi_{a}^{\mu}\left(N, v_{i \backslash j}, P_{i \backslash j}\right)=$ $\Pi_{a}^{\mu}\left(N, w_{i \backslash j}, \hat{P}_{i \backslash j}\right)$, and $i$ and $j$ are doubles in $v$, then the merger axiom implies that $\Pi_{a}^{\mu}\left(N, v_{i \backslash j}, P_{i \backslash j}\right)=\Pi_{a}^{\mu}(N, v, P)$. Then $\Pi_{a}^{\mu}(N, v, P)=\Pi_{a}^{\mu}\left(N, v_{i \backslash j}, P_{i \backslash j}\right)=\Pi_{a}^{\mu}\left(N, w_{i \backslash j}, \hat{P}_{i \backslash j}\right)$. And since $\Pi_{a}^{\mu}(N, w, \hat{P})=\Pi_{a}^{\mu}\left(N, w_{i \backslash j}, \hat{P}_{i \backslash j},\right)$, then $\Pi_{a}^{\mu}(N, v, P)=\Pi_{a}^{\mu}(N, w, \hat{P})$.

Thirdly, observe that $\sum_{\substack{P_{s}, P_{t} \in \hat{P} \\ P_{s} \neq P_{t}}}\left|P_{s} \cap P_{t}\right|=r$, then by induction $\Pi_{a}^{\mu}(N, w, \hat{P})$ is determined, then $\Pi_{a}^{\mu}(N, v, P)$ to.

b) Now assume that there is not any $j \neq a$, such that $j \in P_{q} \cap P_{l}$ and $P_{q} \neq P_{l}$. Since $\sum_{\substack{P_{q}, P_{l} \in P \\ P_{q} \neq P_{l}}}\left|P_{q} \cap P_{l}\right| \geq 0$, then it holds that $a \in P_{q} \cap P_{l}$ for some $P_{q}, P_{l} \in P$ and $P_{q} \neq P_{l}$. Hence for all $j \in N \backslash a$, we are in case a), then $\Pi_{j}^{\mu}(N, v, P)$ determined for all $j \neq a$. And since $\Pi^{\mu}$ is efficient, then $\Pi_{a}^{\mu}(N, v, P)$ is also determined.

\section{Conclusion}

Throughout this paper we have considered coalition configuration values instead of coalition structure values. A coalition structure is used when the relationship between players are reflexive, symmetric and transitive, while in a coalition configuration the relations are only reflexive and symmetric. Our main result is to define and characterize a class of share functions for games with coalition configuration.

Coalition configurations can also be seen as an undirected and unweighted graph. Therefore we can in future use our results to model some problems on network theory. It would be good to think that the formation of a coalition has a cost which can depend in the size of the coaliton, and generalize share functions under this hypothesis.

Another direction in which to continue research concerns the time spent by a player with the other members of his coalition. It is clear that, with partitions, a player spends all his time with the other members of his coalition. If coalitions can be overlapping, the time allocated to each coalition can influence values. While France and the USA are partners (they belong to NATO), one might think that France and Germany (they belong to EU) are so more often. This would therefore be an interesting development of our work.

Acknowledgements We are grateful to two anonymous referees for very valuable comments and suggestions.

\section{References}

[1] Albizuri, M.J. (2001). An axiomatization of the modified Banzhaf-Coleman index. International Journal of Game Theory, 30, 167-176. 
[2] Albizuri, M.J., Aurrekoetxea, J. (2006a). Coalition configurations and the Banzhaf index. Social Choice and Welfare, 26, 571-596.

[3] Albizuri, M.J., Aurrekoetxea, J., Zarzuelo, J.M. (2006b). Configuration values: extensions of the coalitional Owen value. Games and Economic Behavior, 57, 1-17.

[4] Amer, R., Carreras, F., Giménez, J.M. (2002). The modified Banzhaf value for games with coalition structure: an axiomatic characterization. Mathematical Social Sciences, 43, 45-54.

[5] Aumann, R.J., Drèze, J.H. (1974). Cooperative games with coalition structures. International Journal of Game Theory, 3, 217-237.

[6] Aumann, R.J., Myerson, R.B. (1988). Endogenous formation of links between players and of coalitions: an application of the Shapley value. In Roth, A.E. (Ed.), The Shapley value: Essays in honor of Lloyd S. Shapley (pp. 175-191). Cambridge: Univ. Press..

[7] Banzhaf, J.F. (1965). Weighted voting doesn't work: a mathematical analysis. Rutgers Law Review, 19, 317343.

[8] Coleman, J.S. (1971). Control of collectivities and the power of a collectivity to act. In Lieberman B. (Ed.), Social Choice (pp. 269-300). New York: Gordon and Breach.

[9] Deegan, J., Packel, E.W. (1979). A new index of power for simple n-person games. International Journal of Game Theory, 7, 113-123.

[10] Dubey, P., Shapley, L.S. (1979). Mathematical properties of the Banzhaf power index. Mathematics of Operations Research, 4, 99-131.

[11] Haller, H. (1994). Collusion properties of values. International Journal of Game Theory, 23, 261-281.

[12] Hamiache, G. (1999). A new axiomatization of the Owen value for games with coalition structures. Mathematical Social Sciences, 37, 281-305.

[13] Lehrer, E. (1988). An axiomatization of the Banzhaf value. International Journal of Game Theory, 17, 89-99.

[14] Owen, G. (1975). Multilinear extensions and the Banzhaf value. Naval Research Logistics Quarterly, 22, 741750 .

[15] Owen, G. (1977). Values of games with a priori unions. In Henn, R., Moeschlin, O. (Eds.), Essays in mathematical economics and game theory (pp. 76-88). Berlin: Springer-Verlag.

[16] Owen, G. (1981). Modification of the Banzhaf-Coleman index for games with a priori unions. In Holler, M.J. (Ed.), Power, voting and voting power (pp. 232-238). Wurzburg: Physica-Verlag.

[17] Penrose, L.S. (1946). The elementary statistics of majority voting. Journal of the Royal Statistical Society, 109, 53-57.

[18] Shapley, L.S. (1953). A value for n-person games. In Kuhn, H.W., Tucker, A.W. (Eds.), Contributions to the theory of games II. In Annals of Mathematics Studies, vol. 28. Princeton Univ. Press, 307-317.

[19] van der Laan, G., van den Brink, R. (1998). Axiomatization of a class of share functions for N-person games. Theory and Decision, 44, 117-148.

[20] van der Laan, G., van den Brink, R. (2002). A Banzhaf share function for cooperative games in coalition structure. Theory and Decision, 53, 61-86.

[21] van den Brink, R., van der Laan, G., (2005). A class of consistent share functions for games in coalition structure. Games and Economic Behavior, 51, 193-212. 